\title{
Configurations of an Articulated Arm and Singularities of Special Multi-Flags
}

\author{
Fernand PELLETIER ${ }^{\dagger}$ and Mayada SLAYMAN ${ }^{\ddagger}$ \\ † Université de Savoie, Laboratoire de Mathématiques (LAMA), \\ Campus Scientifique, 73376 Le Bourget-du-Lac Cedex, France \\ E-mail: pelletier@univ-savoie.fr \\ $¥$ Department of Mathematical Sciences, Faculty of Sciences II, Lebanese University, Lebanon \\ E-mail: mslayman@ul.edu.lb
}

Received January 29, 2013, in final form May 18, 2014; Published online June 05, 2014 http://dx.doi.org/10.3842/SIGMA.2014.059

\begin{abstract}
P. Mormul has classified the singularities of special multi-flags in terms of "EKR class" encoded by sequences $j_{1}, \ldots, j_{k}$ of integers (see [Singularity Theory Seminar, Warsaw University of Technology, Vol. 8, 2003, 87-100] and [Banach Center Publ., Vol. 65, Polish Acad. Sci., Warsaw, 2004, 157-178]). However, A.L. Castro and R. Montgomery have proposed in [Israel J. Math. 192 (2012), 381-427] a codification of singularities of multiflags by RC and RVT codes. The main results of this paper describe a decomposition of each "EKR" set of depth 1 in terms of RVT codes as well as characterize such a set in terms of configurations of an articulated arm. Indeed, an analogue description for some "EKR" sets of depth 2 is provided. All these results give rise to a complete characterization of all "EKR" sets for $1 \leq k \leq 4$.
\end{abstract}

Key words: special multi-flags distributions; Cartan prolongation; spherical prolongation; articulated arm; rigid bar

2010 Mathematics Subject Classification: 53C17; 58K99; 70B15; 70Q05; 93A30

\section{Introduction and results}

A special multi-flag of step $m \geq 1$ and length $k \geq 1$ is a sequence

$$
\mathbb{D}: D=D_{k} \subset D_{k-1} \subset \cdots \subset D_{j} \subset \cdots \subset D_{1} \subset D_{0}=T M
$$

of distributions of constant rank on a manifold $M$ of dimension $(k+1) m+1$ which satisfies the following conditions (see [8]):

(i) $D_{j-1}=\left[D_{j}, D_{j}\right]$ is the distribution generated by all Lie brackets of sections of $D_{j}$;

(ii) $D_{j}$ is a distribution of constant rank $(k-j+1) m+1$;

(iii) each Cauchy characteristic subdistribution $L\left(D_{j}\right)$ of $D_{j}$ is a subdistribution of constant corank one in each $D_{j+1}$ for $j=1, \ldots, k-1$, and $L\left(D_{k}\right)=0$;

(iv) there exists a completely integrable subdistribution $F \subset D_{1}$ of corank one in $D_{1}$.

(See Section 2.1 for a more precise definition.)

The notion of special multi-flags is described in [9, 11]. Furthermore, for $m \geq 2$, the existence of a completely integrable subdistribution $F$ of $D_{1}$ implies property (iii). This result was first proved in [6] for regular points, and in $[2,14]$ for the general case. When such a distribution $F$ exists, it is then unique (see Remark 2.1). For $m=1$, a special multi-flag is a Goursat flag, and in this case conditions (iii) and (iv) are automatically satisfied but such a distribution $F$ is not unique. One fundamental result on Goursat flags is the existence of locally universal 
Goursat distributions proved by R. Montgomery and M. Zhitomirskii in [7]. More precisely, they define the monster Goursat manifold which is constructed by applying $k$ successive Cartan prolongations. On the other hand, the kinematic system of a car with $k-1$ trailers can be described by an appropriate Goursat distribution $\Delta_{k}$ on $\mathbb{R}^{2} \times\left(\mathbb{S}^{1}\right)^{k}$. Moreover, this Goursat distribution $\Delta_{k}$ is diffeomorphic to the Cartan prolongation of the distribution $\Delta_{k-1}$ on $\mathbb{R}^{2} \times$ $\left(\mathbb{S}^{1}\right)^{k-1}$ (see Appendix D of [7] or Theorem 3.3 of [12]).

A special multi-flag can be considered as a generalization of the notion of Goursat flags and the fundamental result of [2] and [14] is again obtained by Cartan prolongation (see also [9]). Consequently, in this situation, we can build a monster tower by successive Cartan prolongations of $T \mathbb{R}^{m+1}$ (see $[2,3,4,14]$ ):

$$
\cdots \rightarrow P^{k}(m) \rightarrow P^{k-1}(m) \rightarrow \cdots \rightarrow P^{j}(m) \rightarrow \cdots \rightarrow P^{1}(m) \rightarrow P^{0}(m):=\mathbb{R}^{m+1},
$$

where each manifold $P^{j}(m)$ is endowed with a typical distribution $\Delta_{j}$, the Cartan prolongation of $\Delta_{j-1}$ for $1 \leq l \leq k$. In a similar way we can define a natural notion of spherical prolongation, which also gives rise to a tower of sphere bundles (see Section 2.3):

$$
\cdots \rightarrow \hat{P}^{k}(m) \rightarrow \hat{P}^{k-1}(m) \rightarrow \cdots \rightarrow \hat{P}^{j}(m) \rightarrow \cdots \rightarrow \cdots \rightarrow \hat{P}^{1}(m) \rightarrow \hat{P}^{0}(m):=\mathbb{R}^{m+1}
$$

Again, each manifold $\hat{P}^{j}(m)$ is endowed with a typical distribution $\hat{\Delta}_{j}$ which represents the spherical prolongation of $\hat{\Delta}_{j-1}$ for $j \geq 1$. Notice that we have a canonical 2-fold covering:

$$
\hat{P}^{j}(m) \rightarrow P^{j}(m)
$$

for any $j \geq 1$ and $m \geq 2$.

An articulated arm defined in [16] or a system of rigid bars defined in [6] is a kinematic system which can be described by a special multi-flag. More precisely, the configuration space $\mathcal{C}^{k}(m)$ of such a kinematic system is diffeomorphic to $\mathbb{R}^{m+1} \times\left(\mathbb{S}^{m}\right)^{k}$, and this system is characterized by a distribution $\mathcal{D}_{k}$ which generates a special multi-flag of length $k$ (see Section 3.1). Thus we obtain a natural tower of sphere bundles

$$
\mathcal{C}^{k}(m) \rightarrow \mathcal{C}^{k-1}(m) \rightarrow \cdots \rightarrow \mathcal{C}^{j}(m) \rightarrow \mathcal{C}^{j-1}(m) \rightarrow \cdots \rightarrow \mathcal{C}^{1}(m) \rightarrow \mathcal{C}^{0}(m):=\mathbb{R}^{m+1},
$$

where each map $\mathcal{C}^{j}(m) \rightarrow \mathcal{C}^{j-1}(m)$ is a sphere bundle, and each manifold $\mathcal{C}^{j}(m)$ is endowed with a typical distribution $\mathcal{D}_{j}$ associated with the corresponding articulated arm of length $j$ on $\mathbb{R}^{m+1}$ for $1 \leq l \leq k$. Note that by convention, $\mathcal{C}^{0}(m)$ represents the space $\mathbb{R}^{m+1}$ endowed with the distribution $\mathcal{D}_{0}=T \mathbb{R}^{m+1}$ (see Section 3.2).

In this context, we have the following result ${ }^{1}$ :

Theorem 1 (see Theorems 3.2 and 3.4(2)).

1. Let $\hat{\Delta}_{j}$ be the canonical distribution on $\hat{P}^{j}(m)$ obtained after $j$ successive spherical prolongations. Then for each $m \geq 2$ and $1 \leq j \leq k$, there exists a diffeomorphism $F^{j}$ from $\hat{P}^{j}(m)$ to $\mathcal{C}^{j}(m)$ such that:

(i) $\rho^{j} \circ F^{j}=F^{j-1} \circ \hat{\pi}^{j}$, where $\hat{\pi}^{j}: \hat{P}^{j}(m) \rightarrow \hat{P}^{j-1}(m)$ and $\rho^{j}: \mathcal{C}^{j}(m) \rightarrow \mathcal{C}^{j-1}(m)$ are the canonical projections,

(ii) $F_{*}^{j}\left(\hat{\Delta}_{j}\right)=\mathcal{D}_{j}$

\footnotetext{
${ }^{1}$ The reader can find this result in [13] with a summarized proof. As all the arguments used to show this theorem are also essential for proving Theorem 2 and 3 below, thus in this paper we give a complete proof of this result.
} 
2. The commutative diagrams

$$
\begin{aligned}
& \hat{P}^{k}(m) \rightarrow \hat{P}^{k-1}(m) \rightarrow \cdots \rightarrow \hat{P}^{1}(m) \rightarrow \hat{P}^{0}(m):=\mathbb{R}^{m+1} \\
& \begin{array}{c}
\downarrow \\
\mathcal{C}^{k}(m) \rightarrow \mathcal{C}^{k-1}(m)
\end{array} \stackrel{\cdots}{\downarrow} \rightarrow \underset{\mathcal{C}^{1}(m)}{\downarrow} \rightarrow \begin{array}{c}
\downarrow \\
\downarrow
\end{array} \\
& \begin{array}{c}
\downarrow \\
P^{k}(m) \rightarrow P^{k-1}(m)
\end{array} \rightarrow \cdots \rightarrow \underset{P^{1}(m)}{\downarrow} \rightarrow P^{0}(m):=\mathbb{R}^{m+1}
\end{aligned}
$$

have the following properties:

(i) in each horizontal tower, the horizontal map between the space number $j$ and the space number $j-1(1 \leq j \leq k)$ is a sphere fibration for the first two lines and a projective space fibration for the last line;

(ii) in each column number $j(1 \leq j \leq k)$, each vertical map between the first two lines is a diffeomorphism which sends the typical distribution over the source space to the typical distribution over the image space, and each vertical map between the last two lines is a two-fold covering which has the same property.

The singularities of special multi-flags were first described by P. Mormul in [8, 9]. This classification was based on a generalization of Cartan prolongation, and on some "operation" denoted $\mathbf{j}$ which produces a new $(m+1)$-distribution from the previous one. In this way, P. Mormul constructs a coding system which labels the singularity classes of germs of special multi-flags which he calls "extended Kumpera-Ruiz singularity classes of multi-flags" - in short "EKR classes" - (for more details see Section 5.1). An EKR class is coded by a sequence $j_{1}, \ldots, j_{k}$ such that $j_{l+1} \leq 1+\max \left\{j_{1}, \ldots, j_{l}\right\}$. The integer $\max \left\{j_{1}, \ldots, j_{k}\right\}-1$ is called the depth of the EKR class.

Recently, A.L. Castro and R. Montgomery proposed, in [4], a codification of singularities of multi-flags founded on the tower of projective bundles (1.1) using RC and RVT codes. This codification gives rise to a new classification of the singularities of special multi-flags in terms of RVT classes. More precisely, in tower (1.1) one can define sub-towers by taking the tower of Cartan prolongation of any fiber of $P^{j}(m) \rightarrow P^{j-1}(m)$. Therefore, we obtain the "baby monsters" (see [4]). It follows that in each vector space $\Delta_{k}(p) \subset T_{p} P^{k}(m)$ we have a family of "critical" hyperplanes, coming from these sub-towers. We can note, that one of these hyperplanes is the vertical space $V_{p} P^{k}(m)$, i.e. the tangent space on a fiber of $P^{k}(m) \rightarrow P^{k-1}(m)$. A point $p \in P^{k}(m)$ can be written as $p=\left(p_{k-1}, z\right)$, where $p_{k-1} \in P^{k-1}(m)$ and $z$ is a line in $\Delta_{k-1}\left(p_{k-1}\right)$. Therefore, $p$ is called vertical if $z$ is tangent to the fiber at $p_{k-1}$, and tangency if $z$ is not vertical but belongs to one critical hyperplane. Otherwise, $p$ is called regular. Thus, we can affect to $p$ a word composed of letters $\{R, V, T\}$ such that the letter of rank $l$ is either $R, V$ or $T$, depending on whether the projection of $p$ onto $P^{l}(m)$ is regular, vertical, or tangency, respectively.

The main results of this paper are to give a complete description of some EKR sets in terms of RVT codes, as well as an interpretation of such EKR classes and RVT classes, in terms of the configurations of an articulated arm. To make it clear, we need to consider further definitions and notations.

Let $\omega$ be any word in RVT code. We denote by $R^{h}$ or $T^{h}$ a sub-word of $\omega$ which is a sequence of $h$ consecutive letters $R$ or $T$ if $h>0$, and no letter $R$ or $T$, if $h=0$ respectively. Consider now the multi-flag $\mathbb{D}$ on the configuration space $\mathcal{C}^{k}(m)$ associated to an articulated arm $\left(M_{0}, \ldots, M_{k}\right)$ on $\mathbb{R}^{m+1}$ of length $k \geq 1$ (see Section 3.1). The EKR set $\Sigma_{j_{1} \ldots j_{k}}$ is the set of configurations $q \in \mathcal{C}^{k}(m)$ such that the germ of $\mathbb{D}$ at $q$ belongs to the EKR class coded by the sequence $j_{1}, \ldots, j_{k}$. In the same way, the $\mathbf{R V T}$ set $\mathcal{C}_{\omega}$ is the set of configurations $q \in \mathcal{C}^{k}(m)$ whose RVT code is $\omega$. The depth of $\Sigma_{j_{1} \ldots j_{k}}$ is the depth of the EKR class $j_{1} \ldots j_{k}$. Finally, for any EKR class of 1 -depth we will denote by $\left\{i_{1}, \ldots, i_{\nu}\right\}$ the set $\left\{i \in\{1, \ldots, k\} \mid j_{i}=2\right\}$. We then have: 
Theorem 2 (see Theorem 5.2). Let $\left(M_{0}, \ldots, M_{k}\right)$ be an articulated arm.

1. Each EKR set $\Sigma_{j_{1} \ldots j_{k}}$ of depth 1 is an analytic manifold of codimension $\nu$ of $\mathcal{C}^{k}(m)$.

2. A RVT set $\mathcal{C}_{\omega}$ is contained in $\Sigma_{j_{1} \ldots j_{k}}$ if and only if $\omega$ is of type $R^{h_{0}} V T^{l_{1}} R^{h_{1}} \ldots V T^{l_{\nu}} R^{h_{\nu}}$ and each letter $V$ is exactly at rank $i_{1}, \ldots, i_{\nu}$. Such set is an analytic submanifold of $\Sigma_{j_{1} \ldots j_{k}}$ of codimension $l_{1}+\cdots+l_{\nu}$.

3. The EKR set $\Sigma_{j_{1} \ldots j_{k}}$ is the disjoint union of the RVT sets $\mathcal{C}_{\omega}$, where $\omega$ is any word of type $R^{h_{0}} V R^{h} R^{h_{0}} V T^{l_{1}} R^{h_{1}} \ldots V T^{l_{\nu}} R^{h_{\nu}}$.

The following result gives an interpretation of EKR sets of depth 1 in terms of orthogonality properties of an articulated arm:

Theorem 3 (see Theorems 5.2(2) and 5.3). Let $\left(M_{0}, \ldots, M_{k}\right)$ be an articulated arm.

1. A configuration $q \in \mathcal{C}^{k}(m)$ of the articulated arm belongs to the EKR set $\Sigma_{j_{1} \ldots j_{k}}$ of depth 1 if and only if in this configuration the segments $\left[M_{i-2}, M_{i-1}\right]$ and $\left[M_{i-1}, M_{i}\right]$ are orthogonal at $M_{i-1}$ for all $i=i_{1}, \ldots, i_{\nu}$.

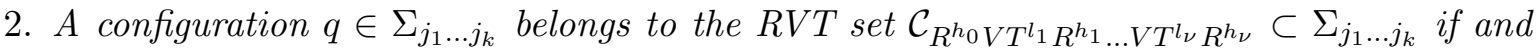
only if, at $q$, the only orthogonality constraint is that each segment $\left[M_{i_{\lambda}+l-1}, M_{i_{\lambda}+l}\right]$ is orthogonal to the direction on $\mathbb{R}^{m+1}$ generated by $\overrightarrow{M_{i_{\lambda}-2} M_{i_{\lambda}-1}}$ for all $l=0, \ldots, l_{\lambda}$ and $\lambda=1, \ldots, \nu$.

This paper is self-contained and organized as follows.

We first recall, in Section 2.1, the context and the essential results about special multi-flags which will be used in this paper. We present a summary on Cartan prolongation and tower of projective bundles in Section 2.2. Spherical prolongations, tower of sphere bundles and their properties are developed in the last Section 2.3.

Section 3 is devoted to the configurations of an articulated arm of length $k \geq 1$ in $\mathbb{R}^{m+1}$. The space $\mathcal{C}^{k}(m)$ of such configurations is presented in Section 3.1. The relation between the tower of sphere bundles (1.2) and the tower (1.3) of configuration spaces $\mathcal{C}^{k}(m)$ is given in Section 3.2. Finally we present the hyperspherical coordinates on $\mathcal{C}^{k}(m)$ in Section 3.3. The reader can find the proof of Theorem 1(1) in Section 3.2 and Theorem 1(2) in Section 3.3.

In Section 4.1, we present a summary of the RC and RVT codes defined in [4], and we adapt these codes to the context of tower of sphere bundles. Section 4.2 gives some interpretations of the property of verticality in terms of configurations of an articulated arm. In an analogous maner, some interpretations of the property of tangency are given in the last Section 4.3.

Section 5 is devoted to the relation between EKR sets of depth 1 and RVT sets. In Section 5.1, we summarize the definition and the results concerning EKR classes based on [8, 9]. Section 5.2 gives a global description of EKR sets in terms of RVT sets. Section 5.3 presents an interpretation of EKR sets (of depth at most 1) and RVT sets in terms of the configurations of an articulated arm. Finally, in Section 5.4 a characterization for some EKR sets of depth 2 in terms of articulated arms is given. In this paragraph, we also give, for $1 \leq k \leq 4$, the decomposition of EKR sets of depth at most 2 in RVT sets and the corresponding interpretation in terms of configurations of an articulated arm. The reader can find the proof of Theorems 2 and 3 in Sections 5.2 and 5.3 respectively.

\section{Preliminaries}

\subsection{Special multi-flags}

A distribution $D$ on a manifold $M$ is an assignment $D: x \in M \mapsto D_{x} \subset T M$ where $D_{x}$ is a linear subspace of the tangent space $T_{x} M$. A local vector field $X$ on $M$ is tangent to $D$ if $X(x)$ belongs 
to $D_{x}$ for all $x$ in the open set on which $X$ is defined. The distribution $D$ is smooth if there exists a set $\mathcal{X}$ of local vector fields such that $D_{x}$ is generated by the set $\{X(x), X \in \mathcal{X}\}$ for all $x$ in some open set $U$. We then say that $D$ is generated by $\mathcal{X}$ on $U$.

In this paper, all distributions are smooth and we denote by $\Gamma(D)$ the set of all local vector fields which are tangent to $D$. A distribution will be called a distribution of constant rank if $D$ defines a subbundle of $T M$. According to [2] and [14], any pair $(M, D)$ where $D$ is a distribution of constant rank on a smooth manifold $M$ is called a differential system. Given two differential systems $(M, D)$ and $(N, \Delta)$ and two points $x \in M$ and $y \in N$, we will say that $(M, D, x)$ is locally equivalent to $(N, \Delta, y)$ if there exists a diffeomorphism $\phi$ from an open neighborhood $U$ of $x$ in $M$ to a neighborhood $V$ of $y$ in $N$ such that $y=\phi(x)$ and $\phi_{*}\left(D_{\mid U}\right)=\Delta_{\mid V}$.

Given a distribution $D^{\prime}$ on $M$ such that $D_{x}^{\prime} \subset D_{x}$ for all $x \in M$, we denote by $\left[D^{\prime}, D\right]$ the distribution generated by the sets $\Gamma(D)$ and $\left\{[X, Y] \mid X \in \Gamma\left(D^{\prime}\right), Y \in \Gamma(D)\right\}$. The Lie square of a distribution $D$ is the distribution $D^{2}:=[D, D]$. The Cauchy characteristic distribution $L(D)$ of a distribution $D$ is the distribution generated by the set of vector fields $\{X \in \Gamma(D) \mid[X, Y] \in$ $D, \forall Y \in \Gamma(D)\}$. If $L(D)$ defines a distribution of constant rank, then it is an integrable distribution.

A special multi-flag of step $m \geq 2$ and length $k \geq 1$ is a sequence of distributions

$$
\mathbb{D}: D=D_{k} \subset D_{k-1} \subset \cdots \subset D_{j} \subset \cdots \subset D_{1} \subset D_{0}=T M
$$

all of constant rank on a manifold $M$ of dimension $(k+1) m+1$ which fulfills the following conditions (see [8]):

(i) $D_{j-1}=\left(D_{j}\right)^{2}$,

(ii) $D_{j}$ is a distribution of constant rank $(k-j+1) m+1$,

(iii) each Cauchy characteristic subdistribution $L\left(D_{j}\right)$ of $D_{j}$ is of constant corank one in each $D_{j+1}$, for $j=1, \ldots, k-1$, and $L\left(D_{k}\right)=0$,

(iv) there exists a completely integrable subdistribution $F \subset D_{1}$ of corank one in $D_{1}$.

In the sequel, a flag $\mathbb{D}$ which satisfies conditions (i) and (ii) without conditions (iii) and (iv) will just be called a multi-flag of step $m$ and we say that $\mathbb{D}$ is generated by $D$.

The necessary and sufficient condition for a multi-flag to be a special multi-flag is given by the following result (see [2, Proposition 1.3] and [14, Theorem 6.2]):

Theorem 2.1 (see $[2,14]$ ). For $k \geq 2$ and $m \geq 1$ consider a multi-flag of step $m$ :

$$
\mathbb{D}: D=D_{k} \subset D_{k-1} \subset \cdots \subset D_{j} \subset \cdots \subset D_{1} \subset D_{0}=T M \text {. }
$$

$\mathbb{D}$ is a special multi-flag if and only if there exists a completely integrable subbundle $F$ of $D_{1}$ of corank 1. Moreover, if the subbundle F exists, then it is unique.

Remark 2.1. The existence of the subbundle $F$ in the previous theorem is crucial and is uniquely determined by the distribution $D_{1}$. In fact, for any subbundle $\mathcal{D}$ of $T M$, the subbundle $F$ of $\mathcal{D}$ was firstly defined by Kumpera and Rubin in [5]. Let us give some details about this fundamental fact (all the following affirmations are proved in $[1,5]$ ).

Let $\mathcal{D}^{\perp} \subset T^{*} M$ be the annihilating Pfaffian system of $\mathcal{D}$, the polar system $\operatorname{Pol}\left(\mathcal{D}^{\perp}\right)$ of the Pfaffian system $(\mathcal{D})^{\perp}$ is defined by

$$
\operatorname{Pol}\left(\mathcal{D}^{\perp}\right)(x)=\left\{\alpha \in T_{x}^{*} M / \mathcal{D}^{\perp}(x) \mid \alpha \wedge \delta \omega=0, \forall \omega \in \mathcal{D}^{\perp}\right\}
$$

where $\delta: \mathcal{D}^{\perp} \rightarrow \Lambda^{2}\left(T^{*} M / \mathcal{D}^{\perp}\right)$ is the Martinet tensor characterized by $\delta \omega=d \omega\left(\bmod \mathcal{D}^{\perp}\right)$. Then, the covariant system $\widehat{\mathcal{D}^{\perp}}$ associated with $\mathcal{D}^{\perp}$ is $q^{-1}\left(\operatorname{Pol}\left(\mathcal{D}^{\perp}\right)\right) \subset T^{*} M$ where $q: T^{*} M \rightarrow$ 
$T^{*} M / \mathcal{D}^{\perp}$ is the canonical projection. It can be proved that, the announced subdistribution $F \subset \mathcal{D}$ is the distribution whose annihilating Pfaffian system is $\widehat{\mathcal{D}^{\perp}}$ (see [1]). When the corank of $\mathcal{D}$ is at most 2 and if $\mathcal{D}^{2}=T M$, then any such distribution $F$ has corank 1 in $\mathcal{D}$ and $F$ is completely integrable (see $[1,5])$.

According to the previous definition of a special multi-flag, we obtain the following sandwich flag:

$$
\begin{array}{ccccccccc}
D_{k} & \subset & D_{k-1} & \subset \cdots \subset & D_{j} & \subset \cdots \subset & D_{1} \subset D_{0}=T M \\
\cup & & \cup & \cdots & \cup & \cdots & \cup &
\end{array}
$$

All vertical inclusions in this diagram are of codimension one, while all horizontal inclusions are of codimension $m$. From these inclusions, we can extract the following "squares subdiagrams" called "sandwiches", indexed by number $j$ which corresponds to the upper left vertices $D_{j}$ :

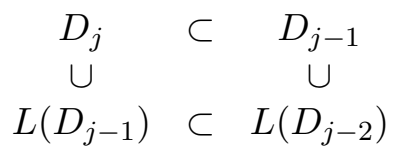

This subdiagram is called a sandwich of rank $j$.

Given a sandwich of rank $j$, and a point $x \in M$, we can look for the relative positions of the $m$ dimensional subspace $L\left(D_{j-2}\right) / L\left(D_{j-1}\right)(x)$ and the 1-dimensional subspace $D_{j} / L\left(D_{j-1}\right)(x)$ in the $(m+1)$-dimensional vector space $D_{j-1} / L\left(D_{j-1}\right)(x)$. One (and only one) of the following situations then occurs:

(i) $L\left(D_{j-2}\right) / L\left(D_{j-1}\right)(x) \oplus D_{j} / L\left(D_{j-1}\right)(x)=D_{j-1} / L\left(D_{j-1}\right)(x)$,

(ii) $D_{j} / L\left(D_{j-1}\right)(x) \subset L\left(D_{j-2}\right) / L\left(D_{j-1}\right)(x)$.

Definition 2.1. A point $x \in M$ is called a Cartan point if $k=1$ or if, for $k \geq 2$, the previous situation (i) is true in each sandwich of rank $j$, for $j=2, \ldots, k$. Otherwise $x$ is called a singular point.

\subsection{Cartan prolongation and tower of projective bundles}

Consider a distribution $D$ of constant rank $m+1$ on a manifold $M$ of dimension $n$. Classically the Grassmannian bundle $G(D, 1, M)$ over $M$ is the set

$$
G(D, 1, M):=\bigcup_{x \in M} P(D(x), 1)
$$

where $P(D(x), 1)$ is the projective space of the vector space $D(x)$. Thus we have a bundle $\pi: G(D, 1, M) \rightarrow M$ whose fiber $\pi^{-1}(x)$ is diffeomorphic to the projective space $\mathbb{R} P^{m}$. The rank one Cartan prolongation of the distribution $D$ is the distribution $D^{(1)}$ defined as follows: given a point $(x, \lambda) \in G(D, 1, M)$, we set

$$
D_{(x, \lambda)}^{(1)}:=d \pi^{-1}(\lambda) \subset T_{(x, \lambda)} G(D, 1, M),
$$

where $\lambda$ is a direction of $D(x)$. Then $D^{(1)}$ is a distribution on $G(D, 1, M)$ of constant rank $m+1$. Let $M$ be a manifold of dimension $m+1$. According to [14], for any $m \geq 2$ and $k \geq 1$ starting with $D=T M$, we obtain inductively a tower of bundles

$$
P^{k}(M) \rightarrow P^{k-1}(M) \rightarrow \cdots \rightarrow P^{1}(M) \rightarrow P^{0}(M):=M
$$


where the sequences $\left(P^{j}(M)\right)_{j=0, \ldots, k}$ and $\left(\Delta_{j}\right)_{j=0, \ldots, k}$ are defined inductively by

$$
\begin{aligned}
& P^{0}(M)=M \quad \text { and } \quad \Delta_{0}=T M, \\
& P^{j}(M)=G\left(\Delta_{j-1}, 1, P^{j-1}(M)\right) \quad \text { and } \quad \Delta_{j}=\left(\Delta_{j-1}\right)^{(1)} \quad \text { for } \quad j=1, \ldots, k .
\end{aligned}
$$

Notice that $P^{j}(M)$ is a manifold of dimension $(j+1) m+1$ for $j=0, \ldots, k$.

In the particular case of $M=\mathbb{R}^{m+1}$, we denote by $P^{j}(m)$ the manifold $P^{j}\left(\mathbb{R}^{m+1}\right)$ for $j=$ $0, \ldots, k$, and we obtain the corresponding tower of bundles:

$$
P^{k}(m) \rightarrow P^{k-1}(m) \rightarrow \cdots \rightarrow P^{1}(m) \rightarrow P^{0}(m):=\mathbb{R}^{m+1} .
$$

Then we have the following result:

Theorem 2.2 (see [14]).

1. On $P^{k}(m)$, the distribution $\Delta_{k}$ generates a special multi-flag of step $m$ and length $k$.

2. Given $x \in M$ and a special multi-flag $\mathbb{D}: D=D_{k} \subset D_{k-1} \subset \cdots \subset D_{j} \subset \cdots \subset D_{1} \subset D_{0}=$ $T M$ of step $m \geq 2$ and length $k \geq 1$, there exists $y \in P^{k}(m)$ for which the differential systems $\left(P^{k}(m), \Delta_{k}, y\right)$ and $(M, D, x)$ are locally equivalent.

Remark 2.2. Theorem 2.2(2) can be found precisely in [14] and is called the "Drapeau theorem". However, according to the definition of a special multi-flag, we can easily deduce this result from the following theorem of [9]:

Theorem 2.3 (see [9]). Suppose that $D$ is a $(m+1)$-dimensional distribution on a $(s+m)$ dimensional manifold $M$ such that the two following conditions are satisfied:

1) $D_{1}=[D, D]$ is a $(2 m+1)$-dimensional distribution on $M$,

2) there exists a 1-codimensional involutive subdistribution $E \subset D$ that preserves $D_{1}$, i.e. $\left[E, D_{1}\right] \subset D_{1}$.

Then $D$ is locally equivalent to the Cartan prolongation $\left(D_{1} / E\right)^{(1)}$ of the reduction $\left(D_{1} / E\right)$ of $D_{1}$ modulo E.

\subsection{Spherical prolongation, Cartan prolongation and tower of sphere bundles}

Consider a distribution $D$ of constant rank $m+1$ on a manifold $M$ of dimension $n$. Choose any Riemannian metric $g$ on $M$, and denote by $S(D, M, g)$ the unit sphere bundle of $D$ associated with the induced Riemannian metric on $D$. Then we obtain a bundle $\hat{\pi}: S(D, M, g) \rightarrow M$ (see Fig. 1).

Consider the antipodal action of $\mathbb{Z}_{2}$ on $S(D, M, g)$. Clearly, the quotient of $S(D, M, g)$ by this action can be identified with $G(D, 1, M)$ and the associated projection $\tau: S(D, M, g) \rightarrow$ $G(D, 1, M)$ is both a bundle morphism over $M$ and a two-fold covering. In particular, $\tau$ is a local diffeomorphism. Consider now the distribution $D^{[1]}$ on $S(D, M, g)$ defined by

$$
D_{(x, \nu)}^{[1]}:=\left\{v \in T_{(x, \nu)} S(D, M, g) \mid d \hat{\pi}(v)=\lambda \nu \text { for some } \lambda \in \mathbb{R}\right\},
$$

where $\nu$ is a unit vector in $D(x)$.

The distribution $D^{[1]}$ is called the rank one spherical prolongation of $(M, D, g)$ (see Fig. 2).

Remark 2.3. The unit sphere bundle associated with $D$ is defined as soon as we fix some Riemannian metric $g_{D}$ on $D$. In this case, the distribution $D^{[1]}$ is well defined and depends only on the Riemannian metric $g_{D}$ on $D$. The spherical prolongation $D^{[1]}$ depends only on the sub-Riemannian structure $\left(M, D, g_{D}\right)$. However, for the sake of simplicity, we always consider Riemannian metrics on $M$. 


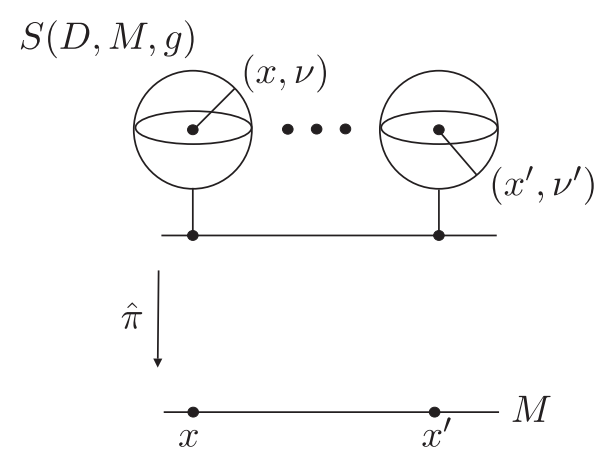

Figure 1. Spherical united bundle.

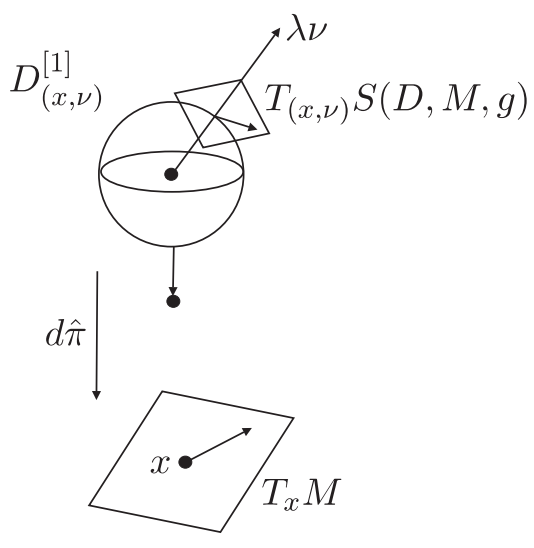

Figure 2. Spherical prolongation on sphere bundle.

Lemma 2.1. With the previous notations we have

(i) $\tau_{*} D^{[1]}=D^{(1)}$, and

(ii) there exists a canonical Riemannian metric $\hat{g}$ on $S(D, M, g)$ which is uniquely defined from the Riemannian metric $g$ on $M$.

Proof. First of all we show (i) locally. Choose a chart domain $U$ over which $D$ is trivial. Fix an orthonormal frame $\left\{e_{0}, \ldots, e_{m}\right\}$ of $D$ over $U$. Without loss of generality, we can assume that $\left.D\right|_{U} \equiv \mathbb{R}^{n} \times \mathbb{R}^{m+1}$ therefore the bundle $\left.S(D, M, g)\right|_{U}$ is isomorphic to $\mathbb{R}^{n} \times \mathbb{S}^{m}$, and $\left.G(D, 1, M)\right|_{U}$ is isomorphic to $\mathbb{R}^{n} \times \mathbb{R} P^{m}$. Then, locally, $\tau: \mathbb{R}^{n} \times \mathbb{S}^{m} \rightarrow \mathbb{R}^{n} \times \mathbb{R} P^{m}$ is the map $(x, \nu) \rightarrow(x,[\nu])$, where $[\nu]$ is the line bundle generated by $\nu$. According to the definition of $D_{(x, \nu)}^{[1]}$ and $D_{\tau(x,[\nu])}^{(1)}$, we have $\tau_{*}\left(D_{(x, \nu)}^{[1]}\right)=D_{\tau(x,[\nu])}^{(1)}$. Since $\tau$ is a local diffeomorphism then (i) is proved locally. The map $\hat{\alpha}: S(D, M, g) \rightarrow S(D, M, g)$ given by $\hat{\alpha}(x, \nu)=(x,-\nu)$ is a diffeomorphism which commutes with $\tau$. According to the definition of $D^{[1]}$, we obtain

$$
\hat{\alpha}_{*}\left(D_{(x, \nu)}^{[1]}\right)=D_{(x,-\nu)}^{[1]} .
$$

This ends the proof of (i).

For (ii), let $\bar{g}$ be the canonical Riemannian metric on $T M$ associated with $g$. Since $S(D, M, g)$ can be considered as a submanifold of $T M$, the metric $\bar{g}$ induces a Riemannian metric $\hat{g}$ on $S(D, M, g)$.

Consider two Riemannian metrics $g_{0}$ and $g_{1}$ on $M$. We denote by $S_{i}(D, M)$ the sphere bundle of $D$ associated with the metric $g_{i}$, and $D_{i}^{[1]}$ the spherical prolongation of $\left(M, D, g_{i}\right)$ for $i=0,1$. 
Lemma 2.2. There exists a canonical isomorphism of sphere bundles $\psi: S_{0}(D, M) \rightarrow S_{1}(D, M)$ such that $\psi_{*}\left(D_{0}^{[1]}\right)=D_{1}^{[1]}$

Proof. The set $D^{0}:=\bigcup_{x \in M}\left[D_{x} \backslash\{0\}\right]$ is an open submanifold of $D \subset T M$ on which we consider the map $\Psi: D^{0} \rightarrow D^{0}$ defined by

$$
\Psi(x, u):=\left(x, \frac{u}{\left[g_{1}(u, u)\right]^{1 / 2}}\right) .
$$

If $\Pi: D \rightarrow M$ is the projection bundle, then for any $(x, u) \in D$ there exists a neighborhood $\hat{U}=\Pi^{-1}(U) \cap D^{0}$ around $(x, u)$ in $D^{0}$ such that $T D_{\mid \hat{U}}^{0}$ can be identified with $\hat{U} \times T_{x} M \times D_{x}$. Then we have:

$$
d \Psi(v, w)=\left(v,-\frac{g_{1}(u, w)}{2\left[g_{1}(u, u)\right]^{3 / 2}}\right) .
$$

$\Psi$ is a diffeomorphism from $D^{0}$ onto itself that commutes with $\Pi$ and sends $S_{0}(D, M)$ to $S_{1}(D, M)$. It follows that the restriction $\psi$ of $\Psi$ to $S_{0}(D, M)$ is a diffeomorphism onto $S_{1}(D, M)$. Moreover, equation (2.2) shows that for any $u$ in the fiber $D_{x}^{0}$ over $x, d \Psi$ maps the linear span $\mathbb{R} u$ onto itself. Thus we have

$$
\psi_{*}\left(D_{0}^{[1]}\right)=D_{1}^{[1]} .
$$

Consider a differential system $\left(M^{\prime}, D^{\prime}\right)$ and $\phi: M \rightarrow M^{\prime}$ an injetive immersion such that $\phi_{*}\left(D_{x}\right) \subset D_{\phi(x)}^{\prime}$ for any $x \in M$. Any Riemanian metric $g^{\prime}$ on $M^{\prime}$, induces, via $\phi$, a Riemannian metric $g$ on $M$, and we can consider the associated spherical prolongation. This generates the following lemma:

Lemma 2.3. With the above notations, the map $\hat{\phi}: S(D, M, g) \rightarrow S\left(D^{\prime}, M^{\prime}, g^{\prime}\right)$ defined by

$$
\hat{\phi}(x, \nu)=\left(\phi(x), d_{x} \phi(\nu)\right)
$$

is a bundle morphism over $\phi$, which is an injective immersion, and $\hat{\phi}$ satisfies the following properties:

(i) $\hat{\phi}(S(D, M, g))=S\left(\phi_{*}(D), \phi(M), g^{\prime}\right)$, and

(ii) $\hat{\phi}_{*}\left(D^{[1]}\right)=\left(\phi_{*}(D)\right)^{[1]} \subset\left(D^{\prime}\right)^{[1]}$.

Moreover, if $\phi$ is a diffeomorphism such that $\phi_{*}(D)=D^{\prime}$, then $\hat{\phi}$ is also a diffeomorphism and we have $\hat{\phi}_{*}\left(D^{[1]}\right)=\left(D^{\prime}\right)^{[1]}$. On the other hand, the Riemannian metric $\hat{\phi}_{*} \hat{g}^{\prime}$ is nothing else but the canonical metric $\hat{g}$ naturally associated with $g$ on $M$.

Proof. First of all it is clear that $\hat{\phi}$ is smooth and is a bundle morphism over $\phi$. Moreover, $\hat{\phi}$ is injective since $\phi$ is an injective immersion.

Note that the tangent space $T_{(x, \nu)} S_{x}$ of the fiber $S_{x}$ over $x$ of $S(D, M, g)$ can be identified with

$$
\left\{v \in D_{x} \mid g(\nu, v)=0\right\}
$$

Now, any $V \in T_{(x, \nu)} S(D, M, g)$ can be written as $V=(u, v)$ with $u \in T_{x} M$ and $v \in T_{(x, \nu)} S_{x}$. Consequently we get

$$
d_{(x, \nu)} \hat{\phi}(u, v)=\left(d_{x} \phi(u), d_{x} \phi(v)\right) .
$$


It follows that $\hat{\phi}$ is an immersion by equation (2.3).

Indeed, since $\phi^{*} g^{\prime}=g$, the differential $d_{x} \phi$ is an isometry on its range, and then $d_{x} \phi\left(S_{x}\right)$ is the fiber over $\phi(x)$ of $S\left(\phi_{*}(D), \phi(M), g^{\prime}\right)$. Thus (i) is proved.

Let $\hat{\pi}: S(D, M, g) \rightarrow M$ and $\hat{\pi}^{\prime}: S\left(D^{\prime}, M^{\prime}, g^{\prime}\right) \rightarrow M^{\prime}$ be the natural projections. Then we have

$$
d \hat{\pi}^{\prime} \circ d \hat{\phi}=d \phi \circ d \hat{\pi}
$$

which yields

$$
\begin{aligned}
& \left\{\hat{\phi}_{*}\left(D^{[1]}\right)\right\}_{\hat{\phi}(x, \nu)}=\left\{d \hat{\phi}(u, v) ;(u, v) \in T_{(x, \nu)} S(D, M, g), d \hat{\pi}(u, v)=\lambda \nu \text { for some } \lambda \in \mathbb{R}\right\} \\
& \quad=\left\{d \hat{\phi}(u, v) ;(u, v) \in T_{(x, \nu)} S(D, M, g), d \phi \circ d \hat{\pi}(u)=d \hat{\pi}^{\prime} \circ d \hat{\phi}(u, v)=\lambda d \phi(\nu), \lambda \in \mathbb{R}\right\} \\
& \quad=\left\{\left(\phi_{*}(D)\right)^{[1]}\right\}_{\hat{\phi}(x, \nu)} .
\end{aligned}
$$

This ends the proof of (ii).

Assume now that $\phi$ is a diffeomorphism such that $\phi_{*}(D)=D^{\prime}$ and let $\psi=\phi^{-1}$. According to the definition of $\hat{\phi}$ and $\hat{\psi}$, it follows trivially that $\hat{\psi} \circ \hat{\phi}=$ Id. Besides, based on the definition of $\left[\phi_{*}(D)\right]^{[1]}$, and since $d_{x} \phi$ is an isomorphism, we must have $\left\{\left(\phi_{*}(D)\right)^{[1]}\right\}_{\hat{\phi}(x, \nu)}=\left\{\left(D^{\prime}\right)^{[1]}\right\}_{\hat{\phi}(x, \nu)}$. Finally, since $\phi$ is an isometry from $(M, g)$ to $\left(M^{\prime}, g^{\prime}\right), d \phi$ is also an isometry for $(T M, \bar{g})$ and $\left(T M^{\prime}, \bar{g}^{\prime}\right)$ if $\bar{g}$ and $\bar{g}^{\prime}$ are the canonical Riemannian metrics on the tangent bundles induced by $g$ and $g^{\prime}$, respectively. As $\hat{g}$ and $\hat{g}^{\prime}$ are the restrictions of $\bar{g}$ and $\bar{g}^{\prime}$ to $S(D, M, g) \subset T M$ and $S\left(D^{\prime}, M^{\prime}, g^{\prime}\right) \subset T M^{\prime}$, respectively, we obtain the last property and the proof of the lemma.

Consequently, as in the context of Cartan prolongation, for any $m \geq 2$ and $k \geq 1$ we can inductively define a tower of sphere bundles (for a fixed choice of the metric $g$ on a manifold $M$ ) as

$$
\hat{P}^{k}(M) \rightarrow \hat{P}^{k-1}(M) \rightarrow \cdots \rightarrow \hat{P}^{j}(M) \rightarrow \hat{P}^{j-1}(M) \rightarrow \cdots \rightarrow \hat{P}^{1}(M) \rightarrow \hat{P}^{0}(M):=M,
$$

where $\hat{P}^{j}(M)$ is a manifold of dimension $(j+1) m+1$ for any $j=0, \ldots, k$, and on each $\hat{P}^{j}(M)$ we have a canonical distribution $\hat{\Delta}_{j}$ and a Riemannian metric $g_{j}$. All these data are inductively defined by:

- $g_{0}=g$ is a given Riemannian metric on $\hat{P}^{0}(M)=M, \hat{\Delta}_{0}=T M$,

- for $1 \leq j \leq k$ :

○ $\hat{P}^{j}(M)=S\left(\hat{\Delta}_{j-1}, \hat{P}^{j-1}(M), g_{j-1}\right)$,

○ $\hat{\Delta}_{j}=\left(\hat{\Delta}_{j-1}\right)^{[1]}$,

○ $g_{j}$ is the Riemannian metric $\hat{g}_{j-1}$ on $S\left(\hat{\Delta}_{j-1}, \hat{P}^{j-1}(M), g_{j-1}\right)$ associated with $g_{j-1}$.

Note that, if $g^{\prime}$ is another Riemannian metric on $M$, according to Lemmas 2.2 and 2.3, we construct, by induction, a family of diffeomorphisms $\psi^{j}$ such that, if

$$
\hat{P}^{\prime k}(M) \rightarrow \hat{P}^{\prime k-1}(M) \rightarrow \cdots \rightarrow \hat{P}^{\prime 1}(M) \rightarrow \hat{P}^{\prime 0}(M):=M
$$

is the tower of sphere bundles associated with the chosen metric $g^{\prime}$ on $M$ we obtain, for all $j=0, \ldots, k$ :

- $\psi^{j}\left(\hat{P}^{j}(M)\right)=\hat{P}^{\prime j}(M)$,

- $\psi^{j}$ is fiber preserving,

- $\psi_{*}^{j}\left(\hat{\Delta}_{j}\right)=\hat{\Delta}_{j}^{\prime}$. 


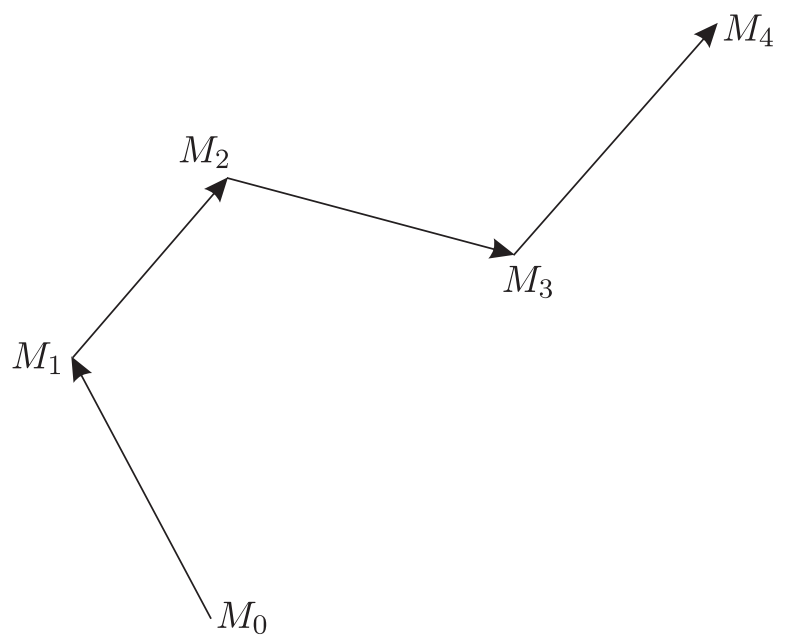

Figure 3. Articulated arm of length 4.

Therefore, the properties which characterize the tower (2.4) are independent of the choice of the Riemannian metric $g$ on $M$.

For the sake of simplicity we write $\hat{P}^{j}(m):=\hat{P}^{j}\left(\mathbb{R}^{m+1}\right)$ for any $j \in \mathbb{N}$. From Theorem 2.2 and Lemma 2.1 we can deduce the following result:

Theorem 2.4. Consider the tower of sphere bundles

$$
\hat{P}^{k}(m) \rightarrow \hat{P}^{k-1}(m) \rightarrow \cdots \rightarrow \hat{P}^{j}(m) \rightarrow \hat{P}^{j-1}(m) \rightarrow \cdots \rightarrow \hat{P}^{1}(m) \rightarrow \hat{P}^{0}(m):=\mathbb{R}^{m+1}
$$

associated with the canonical metric on $\mathbb{R}^{m+1}$. Then the following properties hold:

1. There exists a canonical two-fold covering $\tau^{j}: \hat{P}^{j}(m) \rightarrow P^{j}(m)$ such that $\tau^{j}\left(\hat{\Delta}_{j}\right)=\Delta_{j}$ for all $j=1, \ldots, k$.

2. On $\hat{P}^{j}(m)$, the distribution $\hat{\Delta}_{j}$ generates a special multi-flag of step $m$ and length $j$, for all $j=1, \ldots, k$.

3. Let $\mathbb{D}: D=D_{k} \subset D_{k-1} \subset \cdots \subset D_{j} \subset \cdots \subset D_{1} \subset D_{0}=T M$ be a special multi-flag of step $m \geq 2$ and length $k \geq 1$. Then, for any $x \in M$, there exists $y \in \hat{P}^{k}(m)$ for which the differential system $\left(\hat{P}^{k}(m), \hat{\Delta}_{k}, y\right)$ is locally equivalent to the differential system $(M, D, x)$.

This tower (2.5) will be called the spherical tower of special multi-flags of step $k$.

\section{Tower of sphere bundles associated with a kinematic system}

\subsection{A kinematic system for special multi-flags}

We set ourselves in the context of $[6,16]$. Consider, in $\mathbb{R}^{m+1}$, a family of $k$ segments $\left[M_{i}, M_{i+1}\right]$, where $i=0, \ldots, k-1$ and $m \geq 2$, keeping a constant length $l_{i}=1$ between $M_{i}$ and $M_{i+1}$, with articulation at points $M_{i}$, for $i=1, \ldots, k-1$.

Such a system is called a " $k$-bar system" in [6] and an "articulated arm of length $k$ " in [16]. The kinematic evolution of the extremity $M_{0}$, under the constraint that the velocity of each point $M_{i}, i=0, \ldots, k-1$, is colinear with the segment $\left[M_{i}, M_{i+1}\right]$, is completely described in terms of hyperspherical coordinates in [16], whereas results of flatness and controllability for such a system are proved in [6] (see Fig. 3).

A special multi-flag of step $m \geq 2$ and length $k \geq 1$ is associated with this kinematic system as explained in the following. We can decompose $\left(\mathbb{R}^{m+1}\right)^{k+1}$ into the product $\mathbb{R}_{0}^{m+1} \times \cdots \times$ 
$\mathbb{R}_{i}^{m+1} \times \cdots \times \mathbb{R}_{k}^{m+1}$. Let $x_{i}=\left(x_{i}^{1}, \ldots, x_{i}^{m+1}\right)$ be the canonical coordinates in the space $\mathbb{R}_{i}^{m+1}$ which is equipped with its canonical scalar product $\langle\cdot, \cdot\rangle$. The space $\left(\mathbb{R}^{m+1}\right)^{k+1}$ is then equipped with its canonical scalar product too.

Consider an articulated arm of length $k$ denoted by $\left(M_{0}, \ldots, M_{k}\right)$. We define, on $\left(\mathbb{R}^{m+1}\right)^{k+1}$, the vector fields

$$
\mathcal{Z}_{i}=\sum_{r=1}^{m+1}\left(x_{i+1}^{r}-x_{i}^{r}\right) \frac{\partial}{\partial x_{i}^{r}} \quad \text { for } \quad i=0, \ldots, k-1
$$

Based on our previous assumptions, the kinematic evolution of the articulated arm is described by the controlled system

$$
\dot{q}=\sum_{i=0}^{k-1} u_{i} \mathcal{Z}_{i}+\sum_{r=1}^{m+1} u_{n+r} \frac{\partial}{\partial x_{k}^{r}}
$$

with the following constraints: $\left\|x_{i}-x_{i+1}\right\|=1$ for $i=0, \ldots, k-1$ (see [6] or [16]).

For $k \geq 1$, the configuration space $\mathcal{C}^{k}(m)$ is the set

$$
\left\{\left(x_{0}, \ldots, x_{k}\right) \mid \Psi_{i}\left(x_{0}, \ldots, x_{k}\right)=0, \forall i=0, \ldots, k-1\right\},
$$

where $\Psi_{i}\left(x_{0}, \ldots, x_{k}\right)=\left\|x_{i}-x_{i+1}\right\|^{2}-1$ and we set $\mathcal{C}^{0}(m):=\mathbb{R}^{m+1}$.

For $i=0, \ldots, k-1$, the vector field:

$$
\mathcal{N}_{i}=\sum_{r=1}^{m+1}\left(x_{i+1}^{r}-x_{i}^{r}\right)\left(\frac{\partial}{\partial x_{i+1}^{r}}-\frac{\partial}{\partial x_{i}^{r}}\right)
$$

is proportional to the gradient of $\Psi_{i} \cdot{ }^{2}$ It follows that the tangent space $T_{q} \mathcal{C}^{k}(m)$ is the subspace of $T_{q}\left(\mathbb{R}^{m+1}\right)^{k+1}$ which is orthogonal to $\mathcal{N}_{i}(q)$ for $i=0, \ldots, k-1$.

Denote by $\mathcal{E}_{k}$ the distribution generated by the family of vector fields

$$
\left\{\mathcal{Z}_{0}, \ldots, \mathcal{Z}_{k-1}, \frac{\partial}{\partial x_{k}^{1}}, \ldots, \frac{\partial}{\partial x_{k}^{m+1}}\right\} .
$$

Let $\mathcal{D}_{k}$ be the distribution on $\mathcal{C}^{k}(m)$ defined by $\mathcal{D}_{k}(q)=T_{q} \mathcal{C}^{k}(m) \cap \mathcal{E}_{k}$. Thus we have:

Lemma 3.1 (see [16]). $\mathcal{D}_{k}$ is a distribution of constant rank $m+1$ generated by

$$
\begin{aligned}
& \left(x_{1}^{r}-x_{0}^{r}\right) \mathcal{Z}_{0}+\frac{\partial}{\partial x_{1}^{r}} \quad \text { for } \quad r=1, \ldots, m+1 \quad \text { if } k=1, \\
& \left(x_{k}^{r}-x_{k-1}^{r}\right)\left(\sum_{i=0}^{k-2} \prod_{j=i+1}^{k-1} \mathcal{A}_{j} \mathcal{Z}_{i}+\mathcal{Z}_{k-1}\right)+\frac{\partial}{\partial x_{k}^{r}} \quad \text { for } \quad r=1, \ldots, m+1 \text { if } k \geq 2,
\end{aligned}
$$

where $\mathcal{A}_{j}(q)=-\left\langle\mathcal{N}_{j}(q), \mathcal{N}_{j-1}(q)\right\rangle=\left\langle\mathcal{Z}_{j}(q), \mathcal{N}_{j-1}(q)\right\rangle$, for $j=1, \ldots, k-1$.

Notations 3.1. According to notations of Lemma 3.1, we define

- $Y_{1}=\mathcal{Z}_{0}$ and $Y_{0}=0$,

- for $k \geq 2 Y_{k}=\left(\sum_{i=0}^{k-2} \prod_{j=i+1}^{k-1} \mathcal{A}_{j} \mathcal{Z}_{i}\right)+\mathcal{Z}_{k-1}$.

\footnotetext{
${ }^{2}$ In fact, we could use the differential $d \Psi_{i}$ instead of $\mathcal{N}_{i}$; however, this choice is motivated by the use of the projection $\Pi_{k}$ whose kernel is generated by $\left\{\mathcal{N}_{i} ; i=0, \ldots, k-1\right\}$
} 
Remark 3.1. According to the previous notations, the inductive relation holds:

$$
Y_{k}=\mathcal{A}_{k-1} Y_{k-1}+\mathcal{Z}_{k-1}
$$

Consequently the distribution $\mathcal{D}_{k}$ is generated by the family

$$
\left\{\left(x_{k}^{r}-x_{k-1}^{r}\right) Y_{k}+\frac{\partial}{\partial x_{k}^{r}} \mid r=1, \ldots, m+1\right\} .
$$

The properties of $\mathcal{D}_{k}$ are summarized in the following result (see $\left.[6,16]\right)$ :

Theorem 3.1. The distribution $\mathcal{D}_{k}$ on $\mathcal{C}^{k}(m)$ satisfies the following properties:

1) $\mathcal{D}_{k}$ is a distribution of constant rank $m+1$,

2) $\mathcal{D}_{k}$ generates a special multi-flag on $\mathcal{C}^{k}(m)$ of step $m$ and length $k$.

\subsection{Articulated arm and spherical prolongation}

The following canonical tower of sphere bundles

$$
\mathcal{C}^{k}(m) \rightarrow \mathcal{C}^{k-1}(m) \rightarrow \cdots \rightarrow \mathcal{C}^{1}(m) \rightarrow \mathcal{C}^{0}(m):=\mathbb{R}^{m+1}
$$

is associated with an articulated arm on $\mathbb{R}^{m+1}(m \geq 2)$ of length $k \geq 1$, where, for $j=1, \ldots, k$, the projection $\mathcal{C}^{j}(m) \rightarrow \mathcal{C}^{j-1}(m)$ is the restriction of the canonical projection

$$
\begin{array}{ccc}
\mathbb{R}_{0}^{m+1} \times \cdots \times \mathbb{R}_{i}^{m+1} \times \cdots \times \mathbb{R}_{j}^{m+1} & \rightarrow & \mathbb{R}_{0}^{m+1} \times \cdots \times \mathbb{R}_{i}^{m+1} \times \cdots \times \mathbb{R}_{j-1}^{m+1} \\
\left(x_{0}, \ldots, x_{j-1}, x_{j}\right) & \mapsto & \\
& & \left(x_{0}, \ldots, x_{j-1}\right)
\end{array}
$$

According to Theorems 3.1 and 2.4, we know that the differential system $\left(\mathcal{C}^{k}(m), \mathcal{D}_{k}\right)$ ) associated with an articulated arm of length $k$ on $\mathbb{R}^{m+1}$ is locally isomorphic to the canonical differential system $\left(\hat{P}^{k}(m), \hat{\Delta}_{k}\right)$ at some appropriate points. In fact, obtain a stronger result (as stated in Theorem 1(1)) stated as follows:

Theorem 3.2. For each $m \geq 2$ and $1 \leq j \leq k$, there exists a diffeomorphism $F^{j}$ from $\hat{P}^{j}(m)$ on $\mathcal{C}^{j}(m)$ such that:

(i) $\rho^{j} \circ F^{j}=F^{j-1} \circ \hat{\pi}^{j}$, where $\hat{\pi}^{j}: \hat{P}^{j}(m) \rightarrow \hat{P}^{j-1}(m)$ and $\rho^{j}: \mathcal{C}^{j}(m) \rightarrow \mathcal{C}^{j-1}(m)$ are the canonical projections, and

(ii) $F_{*}^{j}\left(\hat{\Delta}_{j}\right)=\mathcal{D}_{j}$.

Therefore, according to Theorems 2.4 and 3.2 we can obtain:

Theorem 3.3. Let $\mathbb{D}: D=D_{k} \subset D_{k-1} \subset \cdots \subset D_{j} \subset \cdots \subset D_{1} \subset D_{0}=T M$ be a special multiflag of step $m \geq 2$ and length $k \geq 1$. Then, for any $x \in M$, there exists $y \in \mathcal{C}^{k}(m)$ for which the differential system $\left(\mathcal{C}^{k}(m), \mathcal{D}_{k}, y\right)$ is locally equivalent to the differential system $(M, D, x)$.

The end of this subsection is devoted to the proof of Theorem 3.2 and thus the proof of Theorem 1(1). Before doing so, we need some auxiliary results.

Lemma 3.2. For $k \geq 1$, consider the natural decomposition:

$$
\left.\left[T\left(\mathbb{R}^{m+1}\right)^{k+1}\right]\right|_{\mathcal{C}^{k}(m)}=T \mathcal{C}^{k}(m) \oplus\left[T \mathcal{C}^{k}(m)\right]^{\perp} \quad \text { on } \quad \mathcal{C}^{k}(m)
$$

where $\left[\operatorname{TC}^{k}(m)\right]^{\perp}$ is the orthogonal of $T \mathcal{C}^{k}(m)$, and denote by $\Pi_{k}$ the orthogonal projection of $\left[T\left(\mathbb{R}^{m+1}\right)^{k+1}\right]_{\mid \mathcal{C}^{k}(m)}$ onto $T \mathcal{C}^{k}(m)$. Finally, denote by $\mathcal{L}_{k}$ the vertical bundle defined by the natural fibration of $\mathcal{C}^{k}(m)$ over $\mathcal{C}^{k-1}(m)$. We then obtain the following: 
1. The family of vector fields $\left\{\Pi_{k}\left(\frac{\partial}{\partial x_{k}^{r}}\right), r=1, \ldots, m+1\right\}$ generates $\mathcal{L}_{k}$.

2. The distribution $\mathcal{D}_{k}$, is generated by $\mathcal{L}_{k}$ and the vector field $X_{k}=Y_{k}+\mathcal{V}_{k}$, where

$$
\mathcal{V}_{k}=\sum_{s=1}^{m+1}\left(x_{k}^{s}-x_{k-1}^{s}\right) \frac{\partial}{\partial x_{k}^{s}}
$$

3. The distribution $\mathcal{D}_{k}$ is also generated by the family of vector fields

$$
\left(x_{k}^{r}-x_{k-1}^{r}\right) X_{k}+\Pi_{k}\left(\frac{\partial}{\partial x_{k}^{r}}\right), \quad r=1, \ldots, m+1 .
$$

Proof. Denote by $\mathfrak{H}_{k}$ the subdistribution of $\mathcal{E}_{k}$ generated by the family of vector fields

$$
\left\{\frac{\partial}{\partial x_{k}^{r}} \mid r=1, \ldots, m+1\right\} \text {. }
$$

It follows that $\mathfrak{H}_{k} \bigcap T \mathcal{C}^{k}(m)$ is a distribution on $\mathcal{C}^{k}(m)$ which is contained in $\mathcal{D}_{k}$. In fact, we have

$$
\mathcal{L}_{k}=\operatorname{ker} d \Psi_{k-1} \bigcap \mathfrak{H}_{k}=\Pi_{k}\left(\mathfrak{H}_{k}\right) .
$$

Therefore, the distribution $\mathcal{L}_{k}$ is spanned by the family of vector fields

$$
\left\{\Pi_{k}\left(\frac{\partial}{\partial x_{k}^{r}}\right) \mid r=1, \ldots, m+1\right\} .
$$

On the other hand, $\mathfrak{H}_{k}$ is the vertical bundle of the canonical projection,

$$
\begin{array}{ccc}
\mathbb{R}_{0}^{m+1} \times \cdots \times \mathbb{R}_{k}^{m+1} & \rightarrow & \mathbb{R}_{0}^{m+1} \times \cdots \times \mathbb{R}_{k-1}^{m+1} \\
\left(x_{0}, \ldots, x_{k-1}, x_{k}\right) & \mapsto & \left(x_{0}, \ldots, x_{k-1}\right)
\end{array}
$$

It follows that $\mathcal{L}_{k}$ is the vertical bundle of the induced projection of $\mathcal{C}^{k}(m)$ onto $\mathcal{C}^{k-1}(m)$. Moreover, the fiber over $q \in C^{k-1}(m)$ of the previous fibration is the unit sphere $S_{q}=$ $\left\{\left(q, x_{k}\right) \mid \Psi_{k-1}\left(q, x_{k}\right)=0\right\}$, which proves (1).

Furthermore, the vector field $\mathcal{V}_{k}=\sum_{s=1}^{m+1}\left(x_{k}^{s}-x_{k-1}^{s}\right) \frac{\partial}{\partial x_{k}^{s}}$ is vertical for the projection (3.3) and is orthogonal to each $S_{q}$. Since $\left\|\mathcal{V}_{k}\right\|=1$, we thus have

$$
\Pi_{k}\left(\frac{\partial}{\partial x_{k}^{r}}\right)=\frac{\partial}{\partial x_{k}^{r}}-\left(x_{k}^{r}-x_{k-1}^{r}\right) \mathcal{V}_{k}
$$

From Remark 3.1 the distribution $\mathcal{D}_{k}$ is generated by the family

$$
\left\{\left(x_{k}^{r}-x_{k-1}^{r}\right) Y_{k}+\frac{\partial}{\partial x_{k}^{r}} \mid r=1, \ldots, m+1\right\} \text {. }
$$

Therefore the vector field $X_{k}=Y_{k}+\sum_{r=1}^{m+1}\left(x_{k}^{r}-x_{k-1}^{r}\right) \frac{\partial}{\partial x_{k}^{r}}$ is tangent to $\mathcal{D}_{k}$, but clearly this vector field is not tangent to $\mathcal{L}_{k}$. Since $\mathcal{D}_{k}$ is a distribution of constant rank $m+1$ and $\mathcal{L}_{k}$ is an (integrable) subdistribution of rank $m$, then $\mathcal{D}_{k}$ is generated by $\mathcal{L}_{k}$ and $X_{k}$, which proves (2).

Finally, according to the relation (3.4), each vector field $\left(x_{k}^{r}-x_{k-1}^{r}\right) Y_{k}+\frac{\partial}{\partial x_{k}^{r}}$ can be written as

$$
\left(x_{k}^{r}-x_{k-1}^{r}\right) X_{k}+\Pi_{k}\left(\frac{\partial}{\partial x_{k}^{r}}\right) \text {. }
$$

Therefore from Lemma 3.1, we obtain (3). 


\section{Proposition 3.1.}

1. There exists a bundle isomorphism $\hat{\Psi}^{k}: \mathcal{D}_{k} \rightarrow \mathcal{C}^{k}(m) \times \mathbb{R}^{m+1}$ over the identity of $\mathcal{C}^{k}(m)$.

2. Let $\gamma_{k}$ be the Riemannian metric on the bundle $\mathcal{D}_{k}$ such that the morphism $\hat{\Psi}^{k}$ is an isometry between $\mathcal{D}_{k}$ and $\mathcal{C}^{k}(m) \times \mathbb{R}^{m+1}$, where the bundle $\mathcal{C}^{k}(m) \times \mathbb{R}^{m+1}$ is given by the canonical Riemannian metric induced by the canonical Euclidean metric on each fiber $\{q\} \times \mathbb{R}^{m+1}$. Then $\hat{\Psi}^{k}$ induces a diffeomorphism $\Psi^{k}: S\left(\mathcal{D}_{k}, \mathcal{C}^{k}(m), \gamma_{k}\right) \rightarrow \mathcal{C}^{k+1}(m)$ which fulfills the following properties:

(i) $\Psi^{k}$ commutes with the canonical projections $S\left(\mathcal{D}_{k}, \mathcal{C}^{k}(m), \gamma_{k}\right) \rightarrow \mathcal{C}^{k}(m)$ and $\mathcal{C}^{k+1}(m)$ $\rightarrow \mathcal{C}^{k}(m)$

(ii) $\Psi_{*}^{k}\left[\left(\mathcal{D}_{k}\right)^{[1]}\right]=\mathcal{D}_{k+1}$.

Proof. From Remark 3.1, the bundle $\mathcal{D}_{k}$ has $m+1$ non-zero global sections

$$
W_{r}=\left(x_{k}^{r}-x_{k-1}^{r}\right) Y_{k}+\frac{\partial}{\partial x_{k}^{r}} \quad \text { for } \quad r=1, \ldots, m+1 .
$$

Thus $\mathcal{D}_{k}$ is a trivial bundle. This global trivialization gives rise to an isomorphism $\hat{\Psi}^{k}: \mathcal{D}_{k} \rightarrow$ $\mathcal{C}^{k}(m) \times \mathbb{R}^{m+1}$ characterized by

$$
\hat{\Psi}^{k}\left(x_{0}, \ldots, x_{k}, \sum_{r=1}^{m+1} \nu^{r} W_{r}\left(x_{0}, \ldots, x_{k}\right)\right)=\left(x_{0}, \ldots, x_{k}, \nu\right),
$$

where $\nu=\left(\nu^{1}, \ldots, \nu^{m+1}\right)$ and so $\hat{\Psi}^{k}$ satisfies point (1).

Put on $\mathcal{D}_{k}$ the Riemannian metric $\gamma_{k}=\hat{\Psi}^{*} g$, where $g$ is the canonical Euclidean metric on the trivial bundle $\mathcal{C}^{k}(m) \times \mathbb{R}^{m+1}$. It follows that the global basis $\left\{W_{r} \mid r=1, \ldots, m+1\right\}$ is an orthonormal family, and then the set $S\left(\mathcal{D}_{k}, \mathcal{C}^{k}(m), \gamma_{k}\right)$ is:

$$
\left\{\left(x_{0}, \ldots, x_{k}, \sum_{r=1}^{m+1} \nu^{r} W_{r}\left(x_{0}, \ldots, x_{k}\right)\right) \mid\left(x_{0}, \ldots, x_{k}\right) \in \mathcal{C}^{k}(m),\left(\nu^{1}\right)^{2}+\cdots+\left(\nu^{m+1}\right)^{2}=1\right\}
$$

and the fiber over $\left(x_{0}, \ldots, x_{k}\right) \in \mathcal{C}^{k}(m)$ is equal to

$$
\left\{\sum_{r=1}^{m+1} \nu^{r} W_{r}\left(x_{0}, \ldots, x_{k}\right) \mid\left(\nu^{1}\right)^{2}+\cdots+\left(\nu^{m+1}\right)^{2}=1\right\} \text {. }
$$

From the choice of the metric $\gamma_{k}$, the restriction $\bar{\Psi}^{k}$ of $\hat{\Psi}^{k}$ to $S\left(\mathcal{D}_{k}, \mathcal{C}^{k}(m), \gamma_{k}\right)$ is then a diffeomorphism onto $\mathcal{C}^{k}(m) \times \mathbb{S}^{m}$. Moreover, by construction, we have the following commutative diagram:

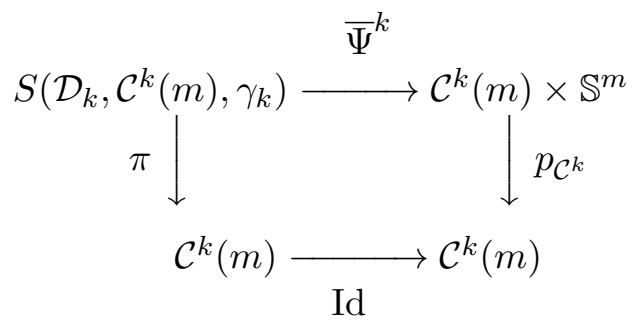

where the vertical maps are the canonical projections. Consider now the map

$$
\widehat{\mathcal{T}}: \mathcal{C}^{k}(m) \times \mathbb{R}^{m+1} \rightarrow \mathcal{C}^{k}(m) \times \mathbb{R}^{m+1}
$$


defined by $\widehat{\mathcal{T}}\left(x_{0}, \ldots, x_{k}, z\right)=\left(x_{0}, \ldots, x_{k}, x_{k}+z\right)$. This map induces a diffeomorphism

$$
\mathcal{T}: \mathcal{C}^{k}(m) \times \mathbb{S}^{m} \rightarrow \mathcal{C}^{k+1}(m)
$$

We set $\Psi^{k}=\mathcal{T} \circ \bar{\Psi}^{k}$ and obtain a diffeomorphism $\Psi^{k}: S\left(\mathcal{D}_{k}, \mathcal{C}^{k}(m), \gamma_{k}\right) \rightarrow \mathcal{C}^{k+1}(m)$ which commutes with the canonical projections of the sphere bundles $\pi: S\left(\mathcal{D}_{k}, \mathcal{C}^{k}(m), \gamma_{k}\right) \rightarrow \mathcal{C}^{k}(m)$ and $\rho^{k+1}: \mathcal{C}^{k+1}(m) \rightarrow \mathcal{C}^{k}(m)$.

Given a vector field $W$ on $\mathcal{C}^{k}(m)$, for any lift $\bar{W}$ of $W$ on $\mathcal{C}^{k+1}(m)$, the vector field $\mathcal{W}=$ $\left(T \Psi^{k}\right)^{-1}(\bar{W})$ is a vector field on the manifold $\mathcal{D}_{k}$ such that $T \pi(\mathcal{W})=W$. Moreover, $T \Psi^{k}$ sends $\operatorname{ker}(T \pi)$ onto $\operatorname{ker}\left(T \rho^{k+1}\right)$. Denote by $\bar{W}_{r}$ the vector field on $\mathcal{C}^{k}(m) \times \mathbb{R}^{m+1}$ defined by

$$
\bar{W}_{r}\left(x_{0}, \ldots, x_{k}, x_{k+1}\right)=\left(x_{k}^{r}-x_{k-1}^{r}\right) Y_{k}+\frac{\partial}{\partial x_{k}^{r}}+\frac{\partial}{\partial x_{k+1}^{r}} .
$$

Notice that $\bar{W}_{r}\left(x_{0}, \ldots, x_{k}, x_{k+1}\right)$ is actually tangent to $\mathcal{C}^{k+1}(m)$, and we have $T p_{\mathcal{C}^{k}}\left(\bar{W}_{r}\right)=W_{r}$.

We set $\mathcal{W}_{r}=\left(T \Psi^{k}\right)^{-1}\left(\bar{W}_{r}\right)$.

Now, given a point $\left(x_{0}, \ldots, x_{k}\right) \in \mathcal{C}^{k}(m)$, the spherical prolongation $\left(\mathcal{D}_{k}\right)^{[1]}$ of $\mathcal{D}_{k}$ at a point $\left(x_{0}, \ldots, x_{k}, \sum_{r=1}^{m+1} \nu^{r} W_{r}\left(x_{0}, \ldots, x_{k}\right)\right)$ is generated by $\sum_{r=1}^{m+1} \nu^{r} \mathcal{W}_{r}\left(x_{0}, \ldots, x_{k}\right)$ and by the tangent space to the fiber through this point. This implies that the space $\Psi_{*}^{k}\left(\left(\mathcal{D}_{k}\right)^{[1]}\right)$ over the point $\left(x_{0}, \ldots, x_{k}, x_{k}+\nu\right)$, is generated by $\sum_{r=1}^{m+1} \nu^{r} \bar{W}_{r}\left(x_{0}, \ldots, x_{k}, x_{k}+\nu\right)$ and by the tangent space of the fiber at this point.

But, according to Remark 3.1, this vector field $\sum_{r=1}^{m+1} \nu^{r} \bar{W}_{r}\left(x_{0}, \ldots, x_{k}, x_{k}+\nu\right)$ can be written as

$$
\sum_{r=1}^{m+1}\left\{\nu^{r}\left(x_{k}^{r}-x_{k-1}^{r}\right) Y_{k}+\nu^{r}\left(\frac{\partial}{\partial x_{k}^{r}}+\frac{\partial}{\partial x_{k+1}^{r}}\right)\right\}=\mathcal{A}_{k} Y_{k}+\mathcal{Z}_{k}+\mathcal{V}_{k+1},
$$

where $x_{k+1}=x_{k}+\nu$. According to (3.1) and Lemma 3.2(2), this last expression is exactly $X_{k+1}$. We deduce that the space $\Psi_{*}^{k}\left(\left(\mathcal{D}_{k}\right)^{[1]}\right)$ is generated, over the point $\left(x_{0}, \ldots, x_{k}, x_{k+1}\right)$, by $X_{k+1}$ and by the tangent space to the fiber at this point. Lemma 3.2(2) implies that $\Psi_{*}^{k}\left(\left(\mathcal{D}_{k}\right)^{[1]}\right)$ and $\mathcal{D}_{k+1}$ coincide at point $\left(x_{0}, \ldots, x_{k}, x_{k+1}\right)$.

Proof of Theorem 3.2. On the one hand, we have $\hat{P}^{0}(m)=\mathcal{C}^{0}(m)=\mathbb{R}^{m+1}$ with $\hat{\Delta}_{0}=\mathcal{D}_{0}=$ $T \mathbb{R}^{m+1}$ and, on the other hand, if $g_{0}$ is the canonical Riemannian metric on $T \mathbb{R}^{m+1}$, we got $P^{1}(m)=S\left(\mathcal{D}_{0}, \mathcal{C}^{0}(m), g_{0}\right)$ with $\hat{\Delta}_{1}=\left(\mathcal{D}_{0}\right)^{[1]}$. Therefore, the result comes from Proposition 3.1 for $k=1$.

Assume that there exists a diffeomorphism $F^{j}: \hat{P}^{j}(m) \rightarrow \mathcal{C}^{j}(m)$ which satisfies properties (i) and (ii) of Theorem 3.2.

From Proposition 3.1, we obtain a diffeomorphism $\Psi^{j}: S\left(\mathcal{D}_{j}, \mathcal{C}^{j}(m), \gamma_{j}\right) \rightarrow \mathcal{C}^{j+1}(m)$ such that $\Psi_{*}\left[\left(\mathcal{D}_{j}\right)^{[1]}\right]=\mathcal{D}_{j+1}$ and satisfying the following commutative diagram

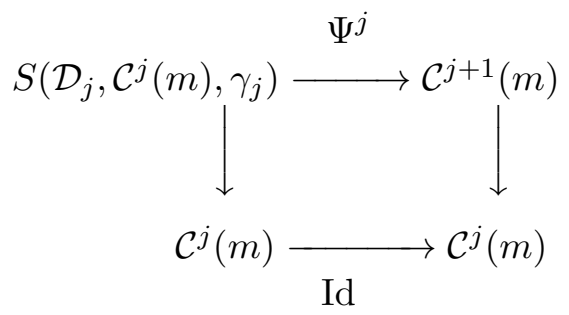


According to previous induction at level $j$, we put on $\hat{P}^{j}(m)$ the Riemannian metric $\bar{\gamma}_{j}=$ $\left(\Psi^{j}\right)^{*}\left(\gamma_{j}\right)$. From Lemma 2.3, we extend $F^{j}: \hat{P}^{j}(m) \rightarrow \mathcal{C}^{j}(m)$ to a diffeomorphism

$$
\hat{\Theta}^{j}: S\left(\hat{\Delta}_{j}, \hat{P}^{j}(m), \bar{\gamma}_{j}\right) \rightarrow S\left(\mathcal{D}_{j}, \mathcal{C}^{j}(m), \gamma_{j}\right)
$$

such that $\hat{\Theta}_{*}^{j}\left[\left(\hat{\Delta}_{j}\right)^{[1]}\right]=\left(\mathcal{D}_{j}\right)^{[1]}$ and satisfying the following commutative diagram

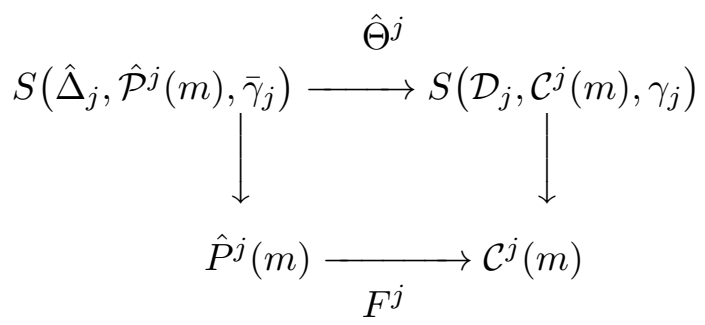

We put on $\hat{P}^{j}(m)$ the Riemannian metric obtained by successive induction on the tower bundle (2.4) (see Section 2.3 just after (2.4)). According to Lemma 2.2, we also obtain a diffeomorphism $\Theta: \hat{P}^{j+1}(m) \rightarrow S\left(\hat{\Delta}_{j}, \hat{P}^{j}(m), \bar{\gamma}_{j}\right)$ such that $\Theta_{*}\left(\hat{\Delta}_{j+1}\right)=\hat{\Delta}_{j}^{[1]}$ and satisfying the following commutative diagram

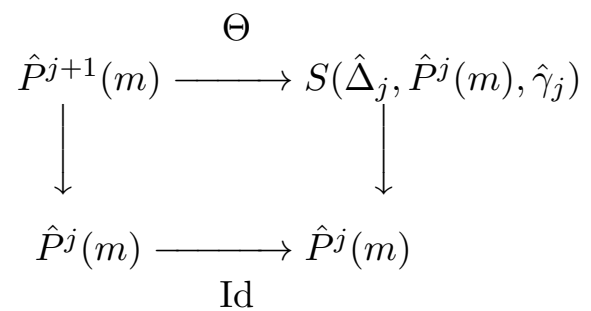

If we juxtapose the three last diagrams, we obtain the required diffeomorphism $F^{j+1}=$ $\Psi^{j} \circ \hat{\Theta}^{j} \circ \Theta$.

Remark 3.2. According to Theorems 2.4 and 3.2, from towers (2.5) and (3.2), we obtain the following diagram in which each vertical map is a 2 -fold covering for $k \geq 1$ :

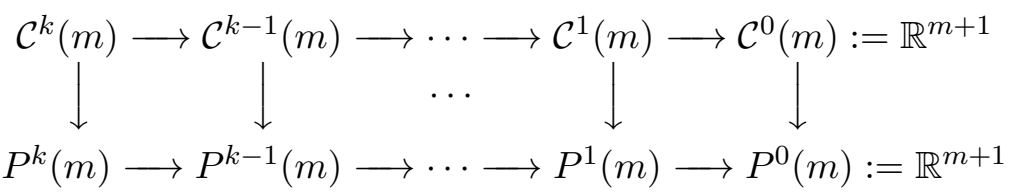

\subsection{Hyperspherical coordinates}

Consider the natural global diffeomorphism $\mathcal{F}^{k}: \mathcal{C}^{k}(m) \rightarrow \mathbb{R}^{m+1} \times\left(\mathbb{S}^{m}\right)^{k}$ given by

$$
\mathcal{F}^{k}\left(x_{0}, x_{1}, \ldots, x_{i}, \ldots, x_{k}\right)=\left(x_{0}, x_{1}-x_{0}, \ldots, x_{i}-x_{i-1}, \ldots, x_{k}-x_{k-1}\right) .
$$

Now, according to Theorem 3.2, the map $\mathcal{F}^{k} \circ F^{k}$ is a global diffeomorphism from $\hat{\mathcal{P}}^{k}(m)$ to $\mathcal{S}^{k}(m)=\mathbb{R}^{m+1} \times\left(\mathbb{S}^{m}\right)^{k}$ and, if $\varrho^{k}: \mathcal{S}^{k} \rightarrow \mathcal{S}^{k-1}$ is the canonical projection, we have the following commutative diagram:

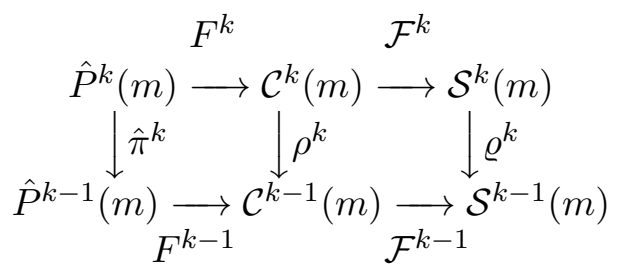


This global chart identifies, each point $q=\left(x_{0}, x_{1}, \ldots, x_{i}, \ldots, x_{k}\right) \in \mathcal{C}^{k}(m)$ with

$$
\zeta=\mathcal{F}^{k}(q)=\left(x_{0}, z_{1}, \ldots, z_{i}, \ldots, z_{k}\right) \in \mathbb{R}^{m+1} \times\left(\mathbb{S}^{m}\right)^{k} .
$$

We will put on each factor $\mathbb{S}^{m}$ the charts given by hyperspherical coordinates in $\mathbb{R}^{m+1}$ defined as usually by the following relations:

$$
\begin{aligned}
& z^{1}=\rho \phi^{1}(\theta)=\rho \sin \theta^{1} \cdots \sin \theta^{m-1} \sin \theta^{m}, \\
& z^{2}=\rho \phi^{2}(\theta)=\rho \sin \theta^{1} \cdots \sin \theta^{m-1} \cos \theta^{m}, \\
& z^{3}=\rho \phi^{3}(\theta)=\rho \sin \theta^{1} \cdots \sin \theta^{m-2} \cos \theta^{m-1}, \\
& \cdots \cdots \cdots \cdots \cdots \cdots \cdots \cdots \cdots \cdots \cdots \cdots \cdots \cdots \cdots \cdots \cdots \cdots \\
& z^{k}=\rho \phi^{k}(\theta)=\rho \sin \theta^{1} \cos \theta^{2} \\
& z^{k+1}=\rho \phi^{k+1}(\theta)=\rho \cos \theta^{1},
\end{aligned}
$$

where $\rho^{2}=\left(z^{1}\right)^{2}+\cdots+\left(z^{k+1}\right)^{2}, 0 \leq \theta^{m} \leq 2 \pi$ and $0 \leq \theta^{j} \leq \pi$ for $1 \leq j \leq m-1$.

We denote by $\hat{\Phi}$ the map from $] 0,+\infty\left[\times[0, \pi] \times \cdots \times[0, \pi] \times[0,2 \pi]\right.$ to $\mathbb{R}^{m+1}$ defined by $\hat{\Phi}(\rho, \theta)=\rho \Phi(\theta)=z$. The jacobian matrix $D \hat{\Phi}$ of $\hat{\Phi}$ is

$$
D \hat{\Phi}=\left(\phi \rho \frac{\partial \phi}{\partial \theta^{1}} \cdots \rho \frac{\partial \phi}{\partial \theta^{m}}\right)
$$

where $\phi$ and $\frac{\partial \phi}{\partial \theta^{j}}$ are the column vectors of components $\left(\phi^{1}, \ldots, \phi^{m+1}\right)$ and $\left(\frac{\partial \phi^{1}}{\partial \theta^{j}}, \ldots, \frac{\partial \phi^{k+1}}{\partial \theta^{j}}\right)$, respectively.

The inverse of this matrix is then the transpose of the matrix

$$
\left(\phi \frac{1}{\rho\left\|\frac{\partial \phi}{\partial \theta^{1}}\right\|} \frac{\partial \phi}{\partial \theta^{1}} \cdots \frac{1}{\rho\left\|\frac{\partial \phi}{\partial \theta^{m}}\right\|} \frac{\partial \phi}{\partial \theta^{m}}\right) .
$$

For $i=1, \ldots, k$, let $\mathbb{S}_{i}$ be the canonical sphere in the $i^{t h}$ factor $\mathbb{R}_{i}^{m+1}$. Given a point $\alpha$ in the sphere $\mathbb{S}_{i}$, there exists hyperspherical coordinates $z_{i}=\hat{\Phi}_{i}\left(\rho_{i}, \theta_{i}\right)=\rho_{i} \Phi_{i}\left(\theta_{i}^{1}, \ldots \theta_{i}^{k}\right)$ defined for $0 \leq \theta_{i}^{k} \leq 2 \pi$ and $0<\theta_{i}^{j}<\pi, j=1, \ldots, m-1$, where $\Phi_{i}(0, \ldots, 0)=\alpha$. Therefore, given a point $\zeta=\left(x_{0}, z_{1}, \ldots, z_{i}, \ldots, z_{k}\right) \in \mathcal{S}^{k}$, we obtain a chart $\mathcal{H}^{k}=\left(\operatorname{Id}-x_{0},\left(\hat{\Phi}_{1}\right)^{-1}, \ldots,\left(\hat{\Phi}_{2}\right)^{-1}, \ldots,\left(\hat{\Phi}_{k}\right)^{-1}\right)$ centered at $\zeta$ such that its restriction to $\rho_{i}=1, i=1, \ldots, k$, induces a chart of $\mathcal{S}^{k}$ (centered at $\zeta)$.

Note that the map $\mathcal{H}^{k}=\left(\operatorname{Id}-x_{0},\left(\hat{\Phi}_{1}\right)^{-1},\left(\hat{\Phi}_{2}\right)^{-1}, \ldots,\left(\hat{\Phi}_{k}\right)^{-1}\right)$ is a hyperspherical chart on $\mathcal{S}^{k}(m)$.

\section{Definition 3.1.}

1. For any $\zeta \in \mathcal{S}^{k}(m)$, every map of type $\mathcal{H}^{k}$ around $\zeta$ is called a hyperspherical chart on $\mathcal{S}^{k}(m)$.

2. For any $q=\left(\mathcal{F}^{k}\right)^{-1}(\zeta)$ in $\mathcal{C}^{k}(m)$, every map of type $\mathcal{H}^{k} \circ \mathcal{F}^{k}$ around $q$ is called a hyperspherical chart on $\mathcal{C}^{k}(m)$.

3. For any $p=\left(\mathcal{F}^{k} \circ F^{k}\right)^{-1}(\zeta)$ on $\hat{\mathcal{P}}^{k}(m)$, every map of type $\mathcal{H}^{k} \circ \mathcal{F}^{k} \circ F^{k}$ around $p$ is called a hyperspherical chart on $\hat{\mathcal{P}}^{k}(m)$.

\section{Notations 3.2.}

- $A_{i}=\sum_{r=1}^{m+1} \phi_{i-1}^{r} \phi_{i}^{r}$ for $i=1, \ldots, k-1$ and $A_{k}=1$, 
- $Z_{0}=\sum_{r=1}^{m+1} \phi_{0}^{r} \frac{\partial}{\partial x_{0}^{r}}$

- $Z_{i}=\sum_{j=1}^{m} B_{i}^{j} \frac{\partial}{\partial \theta_{i-1}^{j}}$ for $i=1, \ldots, k-1$, with $B_{i}^{1}=\sum_{r=1}^{m+1} \frac{\partial \phi_{i-1}^{r}}{\partial \theta_{i-1}^{1}} \phi_{i}^{r}$ for $i=1, \ldots, k-1$, and $B_{i}^{j}=\frac{1}{\left\|\frac{\partial \phi_{i-1}}{\partial \theta_{i-1}^{j}}\right\|} \sum_{r=1}^{m+1} \frac{\partial \phi_{i-1}^{r}}{\partial \theta_{i-1}^{j}} \phi_{i}^{r}$ for $i=1, \ldots, k-1$ and $j=2, \ldots, m$,

- $X_{l}^{i}=\frac{\partial}{\partial \theta_{l}^{i}}$, for $i=1, \ldots, m$ and $0 \leq l \leq k-1$,

- $X_{l}^{0}=\sum_{i=0}^{l} f_{l}^{i} Z_{i}$ for $0 \leq l \leq k-1$, with $f_{l}^{i}=\prod_{j=i+1}^{l} A_{j}$, for $i=0, \ldots, l-1,0 \leq l \leq k-1$ and $f_{l}^{l}=1$.

Remark 3.3. In Lemma 3.1 we already defined a function $\mathcal{A}_{j}(q)=-\left\langle\mathcal{N}_{j}(q), \mathcal{N}_{j-1}(q)\right\rangle$. It is clear that we have the relation $A_{j} \circ\left(\mathcal{H}^{k} \circ \mathcal{F}^{k}\right)=\mathcal{A}_{j}$.

Theorem 1(2) is obtained from (2) of the following result:

Theorem 3.4. For any $k \geq 1$, we have the following properties:

1. In hyperspherical coordinates, on each manifold $\mathcal{S}^{k}(m), \mathcal{C}^{k}(m)$ and $\hat{\mathcal{P}}^{k}(m)$, the corresponding typical distributions $\mathcal{F}_{*}^{k}\left(\mathcal{D}_{k}\right), \mathcal{D}_{k}$ and $\hat{\Delta}_{k}$ are generated by $\left\{X_{k-1}^{0}, X_{k-1}^{1}, \ldots, X_{k-1}^{m}\right\}$, respectively.

2. We have a net of commutative diagrams:

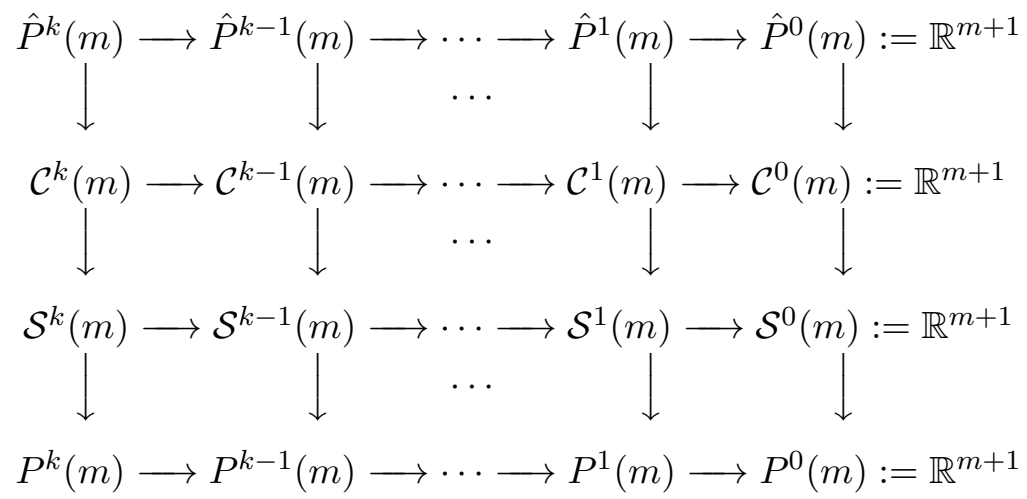

with the following properties:

- in each horizontal tower, the horizontal map between the space number l and the space number $l-1(l \geq 1)$ is a spherical fibration and a projective space fibration in the first three lines and in the last line, respectively.

- In each column number $l$ each vertical map between two consecutive lines among the first three lines is a diffeomorphism which sends the typical distribution over the source space on the typical distribution over the image space, and each vertical map between the two last lines is a two-fold covering which have the same properties.

Proof. In Section 5 of [16], it is proved that, in hyperspherical coordinates, the distribution $F_{*}^{k}\left(\mathcal{D}_{k}\right)$ is precisely generated by $\left\{X_{k-1}^{0}, X_{k-1}^{1}, \ldots, X_{k-1}^{m}\right\}$. According to Theorem 3.2, the diffeomorphism $F^{k}: \hat{\mathcal{P}}^{k}(m) \rightarrow \mathcal{C}^{k}(m)$ sends the distribution $\hat{\Delta}_{k}$ onto $\mathcal{D}_{k}$. This ends the proof of (1). (2) is a consequence of relation (2.5), Theorem 3.2, diagram (3.5) and (1).

Remark 3.4. All manifolds which appear in the towers (3.6) are analytic manifolds and all maps in these towers are also analytic. 


\section{RC and RVT codes and configurations of an articulated arm}

\subsection{RC and RVT codes according to [4]}

In this subsection the theory of $\mathbf{R C}$ and $\mathbf{R V T}$ codes introduced in [4] will be adapted to the context of spherical prolongation.

Consider a distribution $D$ of constant rank on a manifold $M$ fitted with a Riemannian metric $g$. We will denote, indistinctly, by $\mathcal{P}(M, D)$ the sphere bundle $S(M, D, g)$ or the projective bundle $P(M, D)$ and by $D^{\{1\}}$ the spherical prolongation or the Cartan prolongation of $D$ on $\mathcal{P}(M, D)$. Therefore, we have the associated tower of bundles (see (2.1) and (2.4))

$$
\mathcal{P}^{k}(M) \longrightarrow \mathcal{P}^{k-1}(M) \longrightarrow \cdots \longrightarrow \mathcal{P}^{1}(M) \longrightarrow \mathcal{P}^{0}(M):=M,
$$

where each manifold $\mathcal{P}^{j}$ is equipped with a distribution denoted by $\mathfrak{D}_{j}$ such that $\mathfrak{D}_{0}=T M$ and $\mathfrak{D}_{j}=\left(\mathfrak{D}_{j-1}\right)^{\{1\}}$ for $1 \leq j \leq k$. When $M=\mathbb{R}^{m+1}, \mathcal{P}^{k}(m)$ denotes either $\hat{P}^{k}(m)$ or $P^{k}(m)$.

For any $1 \leq j \leq k$, we denote by $\pi^{j}$ the natural projection of $\mathcal{P}^{j}(m)$ onto $\mathcal{P}^{j-1}(m)$. The tangent bundle to the fiber of $\pi^{j}: \mathcal{P}^{j}(m) \rightarrow \mathcal{P}^{j-1}(m)$ is the vertical bundle denoted by $V_{j}$, and at any $p \in \mathcal{P}^{j}(m), V_{j}(p) \subset \mathfrak{D}_{j}(p)$ by construction.

For any $p \in \mathcal{P}^{k-1}(m)$, the fiber $\left(\pi^{k}\right)^{-1}(p)$ is denoted by $S^{k}(p)$. Thus, for such a point $p,(4.1)$, yields a tower of fiber bundles

$$
\mathcal{P}^{l}\left(S^{k}(p)\right) \longrightarrow \mathcal{P}^{l-1}\left(S^{k}(p)\right) \longrightarrow \cdots \longrightarrow \mathcal{P}^{1}\left(S^{k}(p)\right) \longrightarrow \mathcal{P}^{0}\left(S^{k}(p)\right):=S^{k}(p)
$$

for any $1 \leq l \leq k$.

Coming back to our general context, we have again a distribution $\mathfrak{d}_{j}^{k}$ defined inductively on each $\mathcal{P}^{j}\left(S^{k}(p)\right)$, by $\mathfrak{d}_{0}^{k}=V_{k}$ and $\mathfrak{d}_{j}^{k}=\left[\mathfrak{d}_{j-1}^{k}\right]^{\{1\}}$ for $1 \leq j \leq k$. Such a tower will be called a fiber prolongation tower. Of course, we have $\mathcal{P}^{l}\left(S^{k}(p)\right) \subset \mathcal{P}^{k+l}(m)$ and $\mathfrak{d}_{j}^{k}(q)$ is an hyperplane in $\mathfrak{D}_{k+j}(q)$ for any $q \in \mathcal{P}^{j}\left(S^{k}(p)\right)$. In particular, $\mathfrak{d}_{0}^{k}(q)$ is nothing else but the tangent space of $S^{k}(p)$ at $q$.

On the other hand, for any $k>l \geq 0$, let $\pi^{k l}$ be the natural projection of $\mathcal{P}^{k}(m)$ onto $\mathcal{P}^{l}(m)$ given by the composition $\pi^{k} \circ \pi^{k-1} \circ \cdots \circ \pi^{l+1}$. If $p$ is a point in $\mathcal{P}^{k}(M)$, we denote by $p_{l}$ its projection $p_{l}=\pi^{k l}(p) \in \mathcal{P}^{l}(m)$, and we say that $p_{l}$ is under $p_{k}=p$. With these notations, for $k \geq 1$, each point $p_{k} \in \mathcal{P}^{k}(m)$ can be written $\left(p_{k-1}, z\right)$ for some $z \in S^{k}\left(p_{k-1}\right)$.

It follows that, at each level $k \geq 1$ we have the family of hyperplanes $\mathfrak{d}_{j}^{i}(p)$ inside the space $\mathfrak{D}_{k}(p)$, with $i+j=k$. In fact, each $\mathfrak{d}_{j}^{i}(p)$ comes from a fiber prolongation of order $j$ of the tangent space of the fiber $S^{i}\left(p_{i-1}\right)$ for $i=1, \ldots, k$.

Recall that a family $\left(E_{i}\right)_{i \in I}$ of hyperplanes of $\mathbb{R}^{N}$ is in general position if for every finite subset $J$ of $I$ the codimension of the intersection $\bigcap_{i \in J} E_{j}$ is exactly equal to the cardinal of $J$.

Theorem 4.1 (see [4]). The family of hyperplanes $\mathfrak{d}_{j}^{i}(p)$ with $i+j=k$ is in general position inside the space $\mathfrak{D}_{k}(p)$.

Proof. This result is proved in [4] for Cartan prolongation (Theorem 6.1). If $\hat{p}$ is a point in $\hat{P}^{k}(m)$, we denote by $p$ its projection $\tau^{k}(\hat{p})$ in $P^{k}(m)$ (see Theorem 2.4). According to Theorem 2.4, each hyperplane $\mathfrak{d}_{j}^{i}(\hat{p})$ in $\mathfrak{D}_{k}(\hat{p})$ projects, via $\tau^{k}$, onto a hyperplane $\mathfrak{d}_{j}^{i}(p)$ in $\Delta_{k}(p)$ corresponding to the previous process of fiber prolongation in the equation (2.4). The proof in the context of spherical prolongations is then a consequence of Theorem 6.1 in [4]. 
According to $[3,4]$ we have the following definitions:

\section{Definition 4.1.}

1. Any hyperplane $\mathfrak{d}_{j}^{i}(p)$ with $i+j=k$ in the space $\mathfrak{D}_{k}(p)$ is called a critical hyperplane at $p$. A direction $l$ or a vector $v$ in $\mathfrak{D}_{k}(p)$ is called critical if it lies in at least one critical hyperplane. Otherwise $l$ or $v$ is called regular. Moreover, a critical direction $l$ or a vector $v$ in $\mathfrak{D}_{k}(p)$ is called vertical or tangency if the singular hyperplane containing this direction is $V_{k}(p)=\mathfrak{d}_{0}^{k}(p)$ or $\mathfrak{d}_{j}^{i}(\hat{p})$ for $j>0$, respectively.

2. A point $p=\left(p_{k-1}, z\right) \in \mathcal{P}^{k}(m)$ is called regular, critical, vertical or tangency if $z \in$ $\mathfrak{D}_{k-1}\left(p_{k-1}\right)$ is regular, critical, vertical or tangency respectively.

\section{Remark 4.1.}

1. Let $\hat{p} \in \hat{P}^{k}(m)$ and $p=\tau^{k}(\hat{p}) \in P^{k}(m)$. It follows from Theorem 2.4 that $\hat{p}$ is regular, critical, vertical or tangency if and only if $p$ is respectively regular, critical, vertical or tangency. Conversely, for any $p \in P^{k}(m)$, each point in $\tau^{k}(p) \subset \hat{P}^{k}(m)$ has the same previous qualification as $p$.

2. We can consider, inside any fiber prolongations tower given by equation (4.2), a fiber prolongation tower from some fiber of the projection $\mathcal{P}^{l}\left(S^{k}(p)\right) \rightarrow \mathcal{P}^{l-1}\left(S^{k}(p)\right)$ and look for the corresponding critical hyperplane in $\mathfrak{d}_{l}^{k}(q)$. Then such a critical hyperplane is in fact an intersection of type $\mathfrak{d}_{l}^{k}(q) \cap \mathfrak{d}_{j}^{i}(q)$ with $i>k$ and $k+l=i+j$ (see Proposition 6.2 in $[4])$.

3. If a point $p=\left(p_{k-1}, z\right) \in \mathcal{P}^{k}(m)$ is critical, then $z$ may belong to the intersection of several critical hyperplanes and not only to one critical hyperplane.

The $\mathbf{R C}$ code of a point $p \in \mathcal{P}^{k}(m)$ is a word $\sigma=\sigma_{1} \ldots \sigma_{l} \ldots \sigma_{k}$ whose letter $\sigma_{l}$ is $R$ or $C$ if the point $p_{l}$ under $p$ is regular or critical respectively. Note that, by convention, the first letter is always $R$. Let $\sigma$ be the RC code of a point $p \in \mathcal{P}^{k}(m)$. The RVT code of $p$ is a word $\omega=\omega_{1} \ldots \omega_{l} \ldots \omega_{k}$ obtained from $\sigma$ in the following way:

- $\omega_{i}=R$ if $\sigma_{i}=R$,

- $\omega_{i}=V$ if $\sigma_{i}=C$ and the point $p_{i}$ under $p$ is vertical,

- $\omega_{i}=T$ if $\sigma_{i}=C$ and the point $p_{i}$ under $p$ is tangency.

\section{Remark 4.2.}

1. According to Remark 4.1, the RC or RVT code of any point $\hat{p} \in \hat{P}^{k}(m)$ is the same as the RC or RVT code of its projection $p=\tau^{k}(\hat{p}) \in P^{k}(m)$, respectively.

2. The $\mathbf{R C}$ code gives rise to a partition of $\mathcal{P}^{k}(m)$ into $2^{k-1}$ sets of points which have the same RC code $\sigma$. Let $\hat{C}_{\sigma}$ or $C_{\sigma}$ be the set of point $\hat{p} \in \hat{P}^{k}(m)$ or $p \in P^{k}(m)$ whose $\mathbf{R C}$ code is $\sigma$ respectively. Then $\tau^{k}\left(\hat{C}_{\sigma}\right)=C_{\sigma}$ and $\left(\tau^{k}\right)^{-1}\left(C_{\sigma}\right)=\hat{C}_{\sigma}$.

3. Remark 4.1(2) implies that if $p_{i}$ is tangency, then $p_{i}$ must lie in a fiber tower prolongation for some $p_{j}$ under $p_{i}$. Therefore, if $i$ is the level at which the first letter $C$ appears in a $\mathbf{R C}$ word, then the associated point $p_{i}$ must be vertical.

4. Each RC code $\sigma$ generates theoretically $2^{n_{\sigma}} \mathbf{R V T}$ codes $\omega$ if $n_{\sigma}$ is the number of letters $C$ in $\sigma$. However, from (3), a letter $T$ cannot immediately follow a letter $R$ in such a code because each tangency point must lies in a prolongation tower of some point $p_{j}$ under $p_{i}$. Consequently, after a letter $R$, there must appear at least a letter $V$ before any letter $T$. 
5. According to Remark 4.1(3), for any critical point $p=\left(p_{k-1}, z\right) \in \mathcal{P}^{k}(m), z$ may belong to the intersection of several critical hyperplanes. Therefore, the RVT code generated by the $\mathbf{R C}$ code may not be well defined. In this case, when $z$ is not vertical and belongs to only one of them, we need to be more clear in the code about the possible letters "T" that may be written $T_{1}, T_{2}, \ldots, T_{\nu}$. Moreover, much more complicated codification is needed if $z$ belongs to the intersection of several critical hyperplanes. For instance, if this intersection is a line, we can use a codification by letters $L_{1}, L_{2}, \ldots$ as is proposed in [3, 4].

In the RVT code, according to Remark 4.2(5) and [3, 4], we will use the following conventions:

- If $z$ belongs to only one critical hyperplane we will use the letters $V, T_{1}, T_{2}, \ldots$ in the RVT code.

- If $z$ belongs to the intersection between exactly two critical hyperplanes referenced $T_{i}$ and $T_{j}$ we will use letters of type $T_{i j}$ in the RVT code. Moreover, we adopt the following convention: $\mathbf{T}_{\mathbf{0}}$ is always relative to a vertical hyperplane and $\mathbf{T}_{\mathbf{i}}$, for $\mathbf{i}>\mathbf{0}$, is relative to a critical hyperplane which is not vertical.

- More generally, if $z$ belongs to the intersection of exactly $n$ critical hyperplanes referenced $T_{0}=V$ and $T_{i_{1}}, \ldots, T_{i_{n-1}}$ or $T_{i_{1}}, \ldots, T_{i_{n}}$ with $i_{i} \ldots i_{n} \neq 0$ we will use letters of type $T_{0 i_{1} \ldots i_{n}}$ or $T_{i_{1} \ldots i_{n}}$ in the RVT code.

Note that, in a RVT code, a letter $T_{0}$ always means "vertical" and each letter $T_{i}$ with $i>0$ means "tangency".

Definition 4.2. We will say that a word $\omega$ (resp. a class $\mathcal{C}_{\omega}$ ) in RVT code is of depth $d$ if this word $\omega$ contains at least one letter of type $T_{i_{1} \ldots i_{d}}$ with $i_{1} \geq 0$.

For instance, for $m=3$ and $1 \leq k \leq 4$ the different RVT codes which may appear are the following (compare with [4] before Corollary 4.48):

$$
\begin{aligned}
\underline{k=1}: \quad & R, \\
\underline{k=2}: \quad & R R, R V, \\
\underline{k=3}: \quad & R R R, R R V, R V V, R V R, R V T, R T_{0} T_{01}, \\
\underline{k=4}: \quad & R R R R, R R R V, \\
& R R V R, R R V V, R R V T, R R T_{0} T_{01} \\
& R V R R, R V R V, R V V R, R V V V, R V V T, R V T_{0} T_{01}, \\
& R V T R, R V T V, R V T T, R T_{0} T-1 T_{01}, \\
& R T_{0} T_{01} R, R T_{0} T_{01} V, R T_{0} T_{01} T_{1}, R T_{0} T_{01} T_{2}, R T_{0} T_{01} T_{01}, R T_{0} T_{01} T_{02}, R T_{0} T_{01} T_{12} .
\end{aligned}
$$

All the words $R T_{0} T_{01}, R R T_{0} T_{01}, R V T_{0} T_{01}, R T_{0} T_{1} T_{01}, R T_{0} T_{01} R, R T_{0} T_{01} V, R T_{0} T_{01} T_{1}, R T_{0} T_{01} T_{2}$, $R T_{0} T_{01} T_{01}, R T_{0} T_{01} T_{02}$ and $R T_{0} T_{01} T_{12}$ are of depth 2 . The other ones are of depth 1.

\subsection{Vertical points and configurations of articulated arms}

Consider a point $q=\left(x_{0}, \ldots, x_{k}\right) \in \mathcal{C}^{k}(m)$ and let $\hat{p}=\left(F^{k}\right)^{-1}(q) \in \hat{P}^{k}(m)$. For $0 \leq l \leq k$, we denote by $q_{l}=F^{l}\left(\hat{p}_{l}\right) \in \mathcal{C}^{k}(m)$, where $\hat{p}_{l}$ is any point under $\hat{p}$. In fact, according to Theorem 3.2 , we have $q_{l}=\left(x_{0}, \ldots, x_{l}\right)=\rho^{k} \circ \cdots \circ \rho^{l-1}(q)=\rho^{k, l-1}(q)$, where $\rho^{k}: \mathcal{C}^{k}(m) \rightarrow \mathcal{C}^{k-1}(m)$ is the natural projection (see Theorem 3.2). We also say that $q_{l}, l=0, \ldots, k-1$ are points under $q$. Moreover, we can write $q=\left(q_{k-1}, x_{k}\right)$ for $x_{k} \in\left(\rho^{k}\right)^{-1}\left(q_{k-1}\right)$ and, by Theorem $3.2, \hat{p}=\left(F^{k}\right)^{-1}(q)$ is vertical if and only if the direction generated by $x_{k}-x_{k-1}$ is vertical according to the projection $\rho^{k}: \mathcal{C}^{k}(m) \rightarrow \mathcal{C}^{k-1}(m)$.

More generally we can transpose the qualification of points of $\hat{P}^{k}(m)$ onto points of $\mathcal{C}^{k}(m)$ : 
Definition 4.3. Consider a point $q=\left(F^{k}\right)(\hat{p}) \in \mathcal{C}^{k}(m)$.

1. $q$ is called regular, critical, vertical or tangency if $\hat{p}$ is regular, critical, vertical or tangency respectively.

2. The code of $q$ will be the code of the corresponding point $\hat{p}$.

First of all, we have the following characterization of vertical points in $\mathcal{C}^{k}(m)$ :

Proposition 4.1. Fix some point $q \in \mathcal{C}^{k}(m)$.

1. For all $2 \leq l \leq k-1$, we have the following equivalent properties:

(a) Consider the sandwich of rank $l$ :

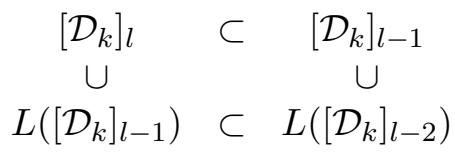

associated with $\mathcal{D}_{k}$. Then $\left[\mathcal{D}_{k}\right]_{l}(q)$ is contained in $L\left(\left[\mathcal{D}_{k}\right]_{l-2}\right)(q)$.

(b) $\mathcal{A}_{l-1}(q)=0$.

(c) The configuration $q$ of the articulated arm $\left(M_{0}, \ldots, M_{k}\right)$ is such that the segments $\left[M_{l-2}, M_{l-1}\right]$ and $\left[M_{l-1}, M_{l}\right]$ are orthogonal at $M_{l-1}$.

(d) $q_{l}$ is vertical.

2. $q$ is a Cartan point if and only if each point $q_{l}$ under $q$ is regular for $1 \leq l \leq k-1$.

Remark 4.3. According to our definition of a singular points (see Definition 2.1), Proposition 4.1 implies that a point $q \in \mathcal{C}^{k}(m)$ is singular if and only if there exists a point $q_{l}$ under $q$ which is vertical.

A consequence of Proposition 4.1 is the following:

\section{Theorem 4.2.}

1. For $k \geq 2$, the set $\mathcal{C}_{S}$ of singular points of $\mathcal{C}^{k}(m)$ is a subanalytic set of codimension 1 . In particular, the set $\mathcal{C}_{C}^{k}(m)=\mathcal{C}^{k}(m) \backslash \mathcal{C}_{S}$ of Cartan points is an open dense set.

2. Let $\omega$ be a word of length $k$ in letters $R$ and $V$ and denote by $\left\{i_{1}, \ldots, i_{\nu}\right\}$ the set of index $\left\{i \in\{1, \ldots, k\} \mid \omega_{i}=V\right\}$. We have the following properties:

(i) The set $\mathcal{C}_{\omega}$ of points $q \in \mathcal{C}^{k}(m)$ whose $\mathbf{R V T}$ code is $\omega$ is an analytic submanifold of $\mathcal{C}^{k}(m)$ of codimension $\nu$.

(ii) The configuration of an articulated arm $\left(M_{0}, \ldots, M_{k}\right)$ belongs to $\mathcal{C}_{\omega}$ if and only if the unique consecutive segments $\left[M_{i-2}, M_{i-1}\right]$ and $\left[M_{i-1}, M_{i}\right]$ which are orthogonal at point $M_{i-1}$ occur for $i=i_{1}, \ldots, i_{\nu}$.

\section{Remark 4.4.}

1. From the definition of a Cartan point (see Definition 2.1) it follows that for $k=1$ all points are Cartan points and the set $\mathcal{C}_{S}$ is empty in this case.

2. Definition 2.1 of a Cartan point is somewhat different from the definition given in $[3,4]$. However Proposition 4.1(2) proves the equivalence of these definitions.

3. The result of Theorem 4.2(1) is well known (see [3, 4, 6, 8, 15]).

4. Theorem 4.2(2) is also proved in [15] but with an another notation for this set. 
The proof of Proposition 4.1 needs the following lemma:

Lemma 4.1. For $2 \leq l \leq k$ consider the sandwich of rank $l$ :

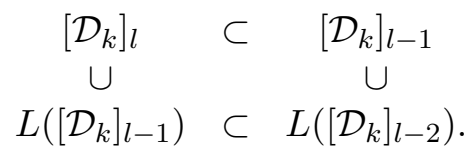

Then $A_{l-1}(q)=0$ if and only if $\left[\mathcal{D}_{k}\right]_{l}(q) \subset L\left(\left[\mathcal{D}_{k}\right]_{l-2}\right)(q)$, and also $q_{l}$ is vertical if and only if $A_{l-1}(q)=0$.

Proof. Let us use Notations 3.2.

By a simple computation (see the proof of Proposition 6.1 in [16]), we conclude that the distribution $\left[\mathcal{D}_{k}\right]_{l}$ of the multi-flag associated with $\mathcal{D}_{k}$ is generated, in hyperspherical coordinates, by the union of the sets $\left\{X_{l-1}^{0}, X_{l-1}^{1}, \ldots, X_{l-1}^{m}\right\}$ and $\left\{X_{i}^{j} \mid j=1, \ldots, m, l-2 \leq i \leq k-1\right\}$, and $L\left(\left[\mathcal{D}_{k}\right]_{l-2}\right)$ is generated by $\left\{X_{i}^{j} \mid j=1, \ldots, m, l-2 \leq i \leq k-1\right\}$.

We also get

$$
X_{l-1}^{0}=A_{l-1} X_{l-2}^{0}+Z_{l-1} .
$$

By construction, each $Z_{l-1}$ belongs to $L\left(\left[\mathcal{D}_{k}\right]_{l-2}\right)$, and hence, if $A_{l-1}(q)=0$, it follows that $\left[\mathcal{D}_{k}\right]_{l}(q) \subset L\left(\left[\mathcal{D}_{k}\right]_{l-2}\right)(q)$. Since $\left\{X_{l-2}^{0}, X_{l-2}^{1}, \ldots, X_{l-2}^{m+1}\right\}$ is a basis of $\mathcal{D}_{l-1}$ at $q_{l-1}$ and $Z_{l-1}$ is a linear combination of $\left\{X_{l-2}^{1}, \ldots, X_{l-2}^{m+1}\right\}$ we then have $X_{l-2}^{0}(q) \neq 0$. Thus, $\left[\mathcal{D}_{k}\right]_{l}(q) \subset$ $L\left(\left[\mathcal{D}_{k}\right]_{l-2}\right)(q)$ if and only if $A_{l-1}(q)=0$. According to Remark 3.3 , this ends the proof of the first equivalence in Lemma 4.1.

Consider now the diffeomorphism $\Psi^{l-1}$ from $S\left(\mathcal{D}_{l-1}, \mathcal{C}^{l-1}(m), \gamma_{l-1}\right)$ onto $\mathcal{C}^{l}(m)$ given in Proposition 3.1. We can write $\Psi^{-1}\left(q_{l}\right)=\left(q_{l-1}, w_{l}\right)$ where $w_{l}$ is a vector of norm 1 in $\mathcal{D}_{l-1}\left(q_{l-1}\right)$. The family $\left\{\left(x_{l-1}^{r}-x_{l-2}^{r}\right) Y_{l-1}+\frac{\partial}{\partial x_{l-1}^{r}} \mid r=1, \ldots, m+1\right\}$ of vector fields (see Lemma 3.2) spans $\mathcal{D}_{l-1}$, and is orthonormal relative to the metric $\gamma_{l-1}$. Therefore, we can write

$$
w_{l}=\sum_{r=1}^{m+1} z_{l}^{r}\left(\left(x_{l-1}^{r}-x_{l_{2}}^{r}\right) Y_{l-1}+\frac{\partial}{\partial x_{l-1}^{r}}\right) .
$$

Moreover, according to this decomposition and from the definition of $\Psi^{l-1}$ in the proof of Proposition 3.1, we have

$$
\Psi^{l-1}\left(q_{l-1}, w_{l}\right)=\left(x_{0}, \ldots, x_{l-1}, x_{l-1}+z_{l}\right),
$$

where $q_{l-1}=\left(x_{0}, \ldots, x_{l-1}\right)$ and $z_{l}=\left(z_{l}^{1}, \ldots, z_{l}^{m+1}\right)$.

Since $\left\{\Pi_{l-1}\left(\frac{\partial}{\partial x_{l-1}^{r}}\right), r=1, \ldots, m+1\right\}$ spans the tangent space to each fiber of the projection $\mathcal{C}^{l-1}(m) \rightarrow \mathcal{C}^{l-2}(m)$, the point $q_{l}$ is vertical if and only if $\sum_{r=1}^{m+1}\left(x_{l}^{r}-x_{l-1}^{r}\right)\left(x_{l-1}^{r}-x_{l-2}^{r}\right)=0$.

But the first member of the previous relation is exactly $\mathcal{A}_{l-1}(q)$. According to Remark 3.3, this ends the proof of the Lemma 4.1.

Proof of Proposition 4.1. Proposition 4.1(1) is a direct consequence of Lemma 4.1.

Now, $q$ is a Cartan point if and only if $\left[\mathcal{D}_{k}\right]_{l}(q)$ is not contained in $L\left(\left[\mathcal{D}_{k}\right]_{l-2}\right)(q)$ for all $2 \leq l \leq k$ (see the end of Section 2.1). We claim that if $q$ is a Cartan point, no point $q_{l}$ under $q$ is vertical. If this was not true, there would exist some point $q_{l}$ under $q$ which is tangency. This would mean that $\hat{p}_{l}=\left(F^{l}\right)^{-1}\left(q_{l}\right) \in P^{l}(m)$ must be tangency. Then, from Remark 4.2(3), there must exist a point $\hat{p}_{j}$ under $\hat{p}_{l}$ which is vertical. Therefore, from Proposition 4.1(1), $q$ cannot be a Cartan point. We conclude that any point $q_{l}$ under $q$ is regular. The converse comes clearly from (1) of the same proposition. 
Proof of Theorem 4.2. If $q$ is singular, from Proposition 4.1(2), there must exist $q_{l}$ under $q$ which is vertical. It follows that the equation of the set $\mathcal{C}_{S}$ is $\prod_{l=1}^{k-1} \mathcal{A}_{l}=0$.

Note that at a point $q$ we have

$$
\frac{\partial \mathcal{A}_{l}}{\partial x_{l+1}^{r}}=x_{l}^{r}-x_{l-1}^{r} \quad \text { for } \quad r=1, \ldots, m+1 .
$$

Taking into account the constraint $\left\|x_{l}-x_{l-1}\right\|^{2}=1$, we must have $\frac{\partial \mathcal{A}_{l}}{\partial x_{l+1}^{r}}(q) \neq 0$ for some $1 \leq r \leq m+1$. According to Remark 3.4, it follows that $\mathcal{C}_{S}$ is a subanalytic subset of $\mathcal{C}^{k}(m)$ of codimension 1 , which ends the proof of (1).

According to the definition of the set $\left\{i_{1}, \ldots, i_{\nu}\right\}$ a point $q$ belongs to $\mathcal{C}_{\omega}$ if and only if each point $q_{i_{1}}, \ldots, q_{i_{\nu}}$ under $q$ is vertical. From Proposition 4.1(b), the equations of $\mathcal{C}(\omega)$ are then

$$
\mathcal{A}_{i}(q)=0 \quad \text { for } \quad i=i_{1}-1, \ldots, i_{\nu}-1,
$$

where each $\mathcal{A}_{l}$ depends only on the variables $x_{l-1}, x_{l}$ and $x_{l+1}$. Thus, since $\frac{\partial \mathcal{A}_{l}}{\partial x_{l+1}^{r}}(q) \neq 0$ for some $1 \leq r \leq m+1$, the equations in (4.3) are independent. According to Remark 3.4, it follows that $\mathcal{C}_{\omega}$ is an analytic submanifold of $\mathcal{C}^{k}(m)$ of codimension $r$.

Theorem 4.2(2) is a direct consequence of Proposition 4.1(c).

\subsection{Tangency points and configurations of articulated arms}

We will prove the fundamental following results for tangency points $q \in \mathcal{C}^{k}(m)$

\section{Theorem 4.3.}

1. Assume that $q \in \mathcal{C}^{k}(m)$ is a tangency point. Then there exists $2 \leq i \leq k-1$ such that the point $q_{i}$ under $q$ is vertical. We define

$$
l=\sup \left\{2 \leq i \leq k-1 \text { such that } q_{i} \text { is vertical } .\right.
$$

Then, if $l<k$, for any $l<j \leq k$, the point $q_{j}$ under $q$ must be tangency.

2. Denote by $R^{h} V T^{l}$ a word of length $h+l+1 \leq k$ in letters $R, T, V$, where $R^{h}$ denotes $h$ consecutive letters $R$ and $T^{l}$ denotes $l$ consecutive letters $T$. Then the set $\mathcal{C}_{R^{h} V T^{l}}$ of points $q \in \mathcal{C}^{h+l+1}(m)$ whose RVT code is $R^{h} V T^{l}$ is an analytic submanifold of $\mathcal{C}^{h+l+1}(m)$ of codimension $l+1$. The fiber of the projection of $\mathcal{C}_{R^{h} V T^{l}}$ onto $\left(\mathcal{C}^{h}(m)\right)_{C}$ over $q_{h} \in\left(\mathcal{C}^{h}(m)\right)_{C}$ is the set $F^{h+l+1}\left(\hat{P}^{l}\left(\hat{S}^{h+1}\left(q_{h}\right)\right)\right)$.

3. To each $q=\left(x_{0}, \ldots, x_{k}\right) \in \mathcal{C}^{k}(m)$ and $0 \leq h<k$ we associate a field of directions $K_{h}(q)$ on $\mathbb{R}^{m+1}$ defined by $K_{h}(q)$ generated by $x_{h+1}-x_{h}$. Given a configuration $q \in \mathcal{C}^{k}(m)$ of an articulated arm $\left(M_{0}, \ldots, M_{k}\right)$, the configuration $q_{h+l+1}$ of the induced articulated arm $\left(M_{0}, \ldots, M_{h+l+1}\right)$ belongs to $\mathcal{C}_{R^{h} V T^{l}}$ if and only if, each segment $\left[M_{h+i}, M_{h+i+1}\right]$ is orthogonal to the direction $K_{h}(q)$ for all $i=0, \ldots, l$ and no other orthogonality constraint.

For the proof we need some intrinsic characterization of $F^{h+l+1}\left(\hat{P}^{l}\left(\hat{S}^{h+1}\left(q_{h}\right)\right)\right)$ in $\mathcal{C}^{h+l+1}(m)$ and the critical hyperplane $F^{h+i+1}\left(\mathfrak{d}_{i}^{h+1}\right)$ in $\mathcal{D}_{h+l+1}$.

Given any vertical point $q=\left(q_{h}, w\right) \in \mathcal{C}^{h+1}(m)$, for $1 \leq h<k$, denote by $\mathcal{C}_{q}^{h+i+1}(m-1)$ the set $F^{h+i}\left(\hat{P}^{i}\left(\hat{S}^{h+1}\left(q_{h}\right)\right)\right)$ of $\mathcal{C}^{h+i+1}(m)$ for $i=1, \ldots, k-h-1$, and call it a critical manifold. For $i=0$ we set $\mathcal{C}_{q}^{h+1}(m-1)=\hat{S}^{h+1}\left(q_{h}\right)$. Let $\delta_{i}^{h+1}$ be the singular hyperplane $F^{h+i+1}\left(\mathfrak{d}_{i}^{h+1}\right) \subset \mathcal{D}_{h+i+1}$ on $\mathcal{C}_{q}^{h+l}(m-1)$, for $i=0, \ldots, k-h-1$. We set $\mathcal{V}_{h+i+1}^{h}=\sum_{s=1}^{m+1}\left(x_{h+1}^{s}-x_{h}^{s}\right) \frac{\partial}{\partial x_{h+i+1}^{s}}$ for $i=0, \ldots, k-h-1$. 
Proposition 4.2. Consider a point $q \in \mathcal{C}^{k}(m)$ such that the point $q_{h+1}=\left(x_{0}, \ldots, x_{h}, x_{h+1}\right)$ under $q$ is vertical. For $i=0, \ldots, k-h-1$, the following properties hold:

(i) the manifold $\mathcal{C}_{q_{h}}^{h+i+1}(m-1)$ is the subset of point $\left(q_{h}, x_{h+1}, \ldots, x_{h+i+1}\right) \in \mathcal{C}^{h+i+1}(m)$ such that:

$$
\left\|x_{h+1}-x_{h}\right\|=1 \quad \text { and } \quad\left\langle x_{j+1}-x_{j}, x_{h+1}-x_{h}\right\rangle=0 \quad \text { for all } j=h+2, \ldots, h+i-1,
$$

(ii) if $i>0$ the vertical space associated with the fibration $\mathcal{C}_{q_{h}}^{h+i+1}(m-1) \rightarrow \mathcal{C}_{q_{h}}^{h+i}(m-1)$ is generated by

$$
\left\{\frac{\partial}{\partial x_{h+i+1}^{r}}-\left(x_{h+1}-x_{h}\right) \mathcal{V}_{h+i+1}^{h}-\left(x_{h+i+1}^{r}-x_{h+i}^{r}\right) \mathcal{V}_{h+i+1} \mid r=1, \ldots, m+1\right\},
$$

(iii) the vector field $X_{h+i+1}$ is tangent to $\mathcal{C}_{q_{h}}^{h+i+1}(m-1)$,

(iv) the distribution $\delta_{i}^{h+1}$ is the intersection between $\mathcal{D}_{h+i+1}$ and the tangent space to $\mathcal{C}_{q_{h}}^{h+i+1}(m-1)$.

Proof. For $i=0$, the set $\mathcal{C}_{q_{h}}^{h+1}(m-1)$ is the sphere $\hat{S}^{h+1}\left(q_{h}\right)$ and the distribution $\delta_{0}^{h+1}$ is the tangent space to this sphere. Moreover, since $q_{h+1}$ is vertical, then $\mathcal{A}_{h}\left(q_{h+1}\right)=0$. Therefore, we have $X_{h+1}=\mathcal{Z}_{h}+\mathcal{V}_{h+1}$ which is tangent to $\hat{S}^{h+1}\left(q_{h}\right)$ in $q_{h+1}$. Thus the properties (i), (iii) and (iv) of Proposition 4.2 are true for $i=0$. Assume now that for all $0 \leq j<i$ these last properties are true. According to our assumption, $\delta_{i-1}^{h+1}$ is generated by the family:

$$
\begin{aligned}
& \left\{X_{h+1}, \frac{\partial}{\partial x_{h+1}^{r}}-\left(x_{h+1}^{r}-x_{h}\right) \mathcal{V}_{h+1} \mid r=1, \ldots, m+1\right\} \text { for } i=1, \\
& \left\{X_{h+i}, \frac{\partial}{\partial x_{h+i}^{r}}-\left(x_{h+1}-x_{h}\right) \mathcal{V}_{h+i}^{h}-\left(x_{h+i}^{r}-x_{h+i-1}\right) \mathcal{V}_{h+i} \mid r=1, \ldots, m+1\right\} \quad \text { for } i>1 .
\end{aligned}
$$

Recall that $\mathcal{D}_{h+i}$ is generated by:

$$
\left\{X_{h+i}, \frac{\partial}{\partial x_{h+i}^{r}}-\left(x_{h+i}^{r}-x_{h+i-1}\right) \mathcal{V}_{h+i} \mid r=1, \ldots, m+1\right\} .
$$

Therefore on $\mathcal{C}_{q_{h}}^{h+i}(m-1)$ the distribution $\delta_{i-1}^{h+1}$ is the intersection between $\mathcal{D}_{h+i}$ and the kernel of the differential form $\sum_{s=1}^{m+1}\left(x_{h+1}^{s}-x_{h}^{s}\right)\left(d x_{h+i}^{s}-d x_{h+i-1}^{s}\right)$.

According to our assumption, we can see that $\delta_{i-1}^{h+1}$ is generated by the family

$$
\left\{U_{r}=\left(x_{h+i}^{r}-x_{h+i-1}^{r}\right) Y_{h+i}+\frac{\partial}{\partial x_{h+i}^{r}}-\left(x_{h+i}^{r}-x_{h+i-1}^{r}\right) \mathcal{V}_{h+i}^{h} \mid r=1, \ldots, m+1\right\} .
$$

According to the proof of Proposition 3.1, a point $\left(q_{h}, x_{h+1}, \ldots, x_{h+i}, \sum_{r=1}^{m+1} \nu^{r} W_{r}\right)$ belongs to the manifold $\delta_{i-1}^{h+1}$ if and only if $\left(q_{h}, x_{h+1}, \ldots, x_{h+i}\right)$ belongs to $\mathcal{C}_{q_{h}}^{h+i}(m-1)$ and $\sum_{r=1}^{m+1} \nu^{r}\left(x_{h+1}^{r}-\right.$ $\left.x_{h}^{r}\right)=0$.

Note that at such a point we have:

$$
\sum_{r=1}^{m+1} \nu^{r} U_{r}=\sum_{r=1}^{m+1} \nu^{r} W_{r}-\sum_{r=1}^{m+1} \nu^{r}\left(x_{h+1}^{r}-x_{h}^{r}\right) \mathcal{V}_{h+1}^{h}=\sum_{r=1}^{m+1} \nu^{r} W_{r}
$$


Consider the submanifold $S\left(\delta_{i-1}^{h+1}, \mathcal{C}_{q_{h}}^{h+i}(m-1), \gamma_{h+i}\right)$ of $S\left(\mathcal{D}_{h+i}, \mathcal{C}^{h+i}(m), \gamma_{h+i}\right)$. According to the proof of Proposition $3.1 \Psi^{h+i}\left(S\left(\delta_{i-1}^{h+1}, \mathcal{C}_{q_{h}}^{h+i}, \gamma_{h+i}\right)\right)$ is a submanifold of $\mathcal{C}^{h+i+1}(m)$ which is fibered on $\mathcal{C}_{q_{h}}^{h+i}(m-1)$. Moreover the restriction of $\Psi^{h+i}$ to $S\left(\delta_{i-1}^{h+1}, \mathcal{C}_{q_{h}}^{h+i}(m-1), \gamma_{h+i}\right)$ commutes with the canonical projections of $\left.S \delta_{i-1}^{h+1}, \mathcal{C}_{q_{h}}^{h+i}(m-1), \gamma_{h+i}\right)$ and $\Psi^{h+i}\left(S\left(\delta_{i-1}^{h+1}, \mathcal{C}_{q_{h}}^{h+i}(m-1), \gamma_{h+i}\right)\right)$ onto $\mathcal{C}_{q_{h}}^{h+i}(m-1)$ respectively.

Now according to $(4.5)$, the manifold $S\left(\delta_{i-1}^{h+1}, \mathcal{C}_{q_{h}}^{h+i}(m-1), \gamma_{h+i}\right)$ is the set of points

$$
\left(q_{h}, x_{h+1}, \ldots, x_{h+i}, \sum_{r=1}^{m=1} \nu^{r} W_{r}\right) \in S\left(\mathcal{D}_{h+i}, \mathcal{C}^{h+i}(m), \gamma_{h+i}\right)
$$

with the following constraints:

$$
\left(q_{h}, x_{h+1}, \ldots, x_{h+i}\right) \in \mathcal{C}_{q_{h}}^{h+i}(m-1), \quad \sum_{r=1}^{m+1}\left(\nu^{r}\right)^{2}=1, \quad \sum_{r=1}^{m+1} \nu^{r}\left(x_{h+1}^{r}-x_{h}^{r}\right)=0 .
$$

Since $\mathcal{C}^{h+i+1}(m)=\mathcal{C}^{h+i}(m) \times \mathbb{S}^{m}$, then $\Psi^{h+i}\left(S\left(\delta_{i-1}^{h+1}, \mathcal{C}_{q_{h}}^{h+i}(m-1), \gamma_{h+i}\right)\right)$ is a submanifold of $\mathcal{C}_{q_{h}}^{h+i}(m-1) \times \mathbb{S}^{m}$ defined by the equation

$$
\sum_{r=1}^{m+1}\left(x_{h+i+1}^{r}-x_{h+i}^{r}\right)\left(x_{h+1}^{r}-x_{h}^{r}\right)=0 .
$$

But from the construction of $F^{h+i+1}, F^{h+i+1}\left(\hat{P}^{i}\left(\hat{S}^{h+1}\left(q_{h}\right)\right)\right.$ is equal to $\Psi^{h+i}\left(S\left(\delta_{i-1}^{h+1}, \mathcal{C}_{q_{h}}^{h+i}(m-\right.\right.$ $\left.1), \gamma_{h+i}\right)$ ), which is precisely the set $\mathcal{C}_{q_{h}}^{h+i+1}(m-1)$. Therefore is proved (i). This implies that the vertical bundle associated with the fibration $\mathcal{C}_{q_{h}}^{h+i+1}(m-1) \rightarrow \mathcal{C}_{q_{h}}^{h+i}(m-1)$ is generated by the family (4.4).

Let $\bar{U}_{r}$ be the vector field on $\mathcal{C}^{h+i}(m) \times \mathbb{R}^{m+1}$ defined by

$$
\bar{U}_{r}=\left(x_{h+i}^{r}-x_{h+i-1}^{r}\right) Y_{h+i}+\frac{\partial}{\partial x_{h+i}^{r}}-\left(x_{h+i}^{r}-x_{h+i-1}^{r}\right) \mathcal{V}_{h+i}^{h}+\frac{\partial}{\partial x_{k+i+1}^{r}} .
$$

The vector field $\bar{U}_{r}\left(x_{0}, \ldots, x_{h+i}, x_{h+i+1}\right)$ is actually tangent to $\mathcal{C}_{q_{h}}^{h+i+1}(m)$ and $\bar{U}_{r}$ projects onto $U_{r}$.

Therefore as in the proof of Proposition 3.1, we set $\mathcal{U}_{r}=\left(T \Psi^{h+i}\right)^{-1}\left(\bar{U}_{r}\right)$. The distribution $\left[\delta_{i-1}^{h+1}\right]^{[1]}$ is generated by the vertical bundle of the fibration $S\left(\delta_{i-1}^{h+1}, \mathcal{C}_{q_{h}}^{h+i}(m-1), \gamma_{h+i}\right) \rightarrow$ $\mathcal{C}_{q_{h}}^{h+i}(m-1)$ and the vector field $\sum_{r=1}^{m+1} \nu^{r} \mathcal{U}_{r}$. According to equation (4.6), the vector field $T \Psi^{h+i}\left(\sum_{r=1}^{m+1} \nu^{r} \mathcal{U}_{r}\right)$ is nothing else but $X_{h+i+1}$ on $\mathcal{C}_{q_{h}}^{h+i+1}(m)$. Moreover, since $\bar{U}_{r}$ is tangent to $\mathcal{C}_{q_{h}}^{h+i+1}(m)$, then $X_{h+i+1}$ is also tangent to $\mathcal{C}_{q_{h}}^{h+i+1}(m)$. Finally the distribution $\Psi_{*}^{h+i}\left(\left[\delta_{i-1}^{h+1}\right]^{[1]}\right)$ is generated by $X_{h+i+1}$ and the vertical bundle of the fibration $\mathcal{C}_{q_{h}}^{h+i+1}(m-1) \rightarrow \mathcal{C}_{q_{h}}^{h+i}(m-1)$. Since this vertical bundle is the intersection between the vertical bundle of the fibration $\mathcal{C}^{k+i+1}(m) \rightarrow$ $\mathcal{C}^{h+i}(m)$ and the tangent space to $\mathcal{C}_{q_{h}}^{h+i+1}(m-1)$ then $\Psi_{*}^{h+i}\left(\left[\delta_{i-1}^{h+1}\right]^{[1]}\right)$ is the intersection between $\mathcal{D}_{h+i+1}$ and the tangent space to $\mathcal{C}_{q_{h}}^{h+i+1}(m-1)$. But according to the definition of the family of distributions $\left\{\delta_{i}^{h+1}\right\}_{i \geq 0}$ and the construction of $F^{h+i+1}$ we get $\Psi_{*}^{h+i}\left(\left[\delta_{i-1}^{h+1}\right]^{[1]}\right)=\delta_{i}^{h+1}$. This ends the proof.

Proof of Theorem 4.3. Fix some tangency point $q \in \mathcal{C}^{k}(m)$. According to Remark 4.2(3), there must exist a vertical point $q_{i}$ under $q$. Let $q_{l}$ be the last vertical point under $q$ then $\mathcal{A}_{l-1}(q)=0$. In fact, given $q_{l-1}=\left(x_{0}, \ldots, x_{l-1}\right)$, this relation characterizes the vertical points 
$q_{l}=\left(x_{0}, \ldots, x_{l-1}, x_{l}\right) \in \mathcal{C}^{l}(m) . F^{l}\left(\hat{S}^{l}\left(q_{l-1}\right)\right)$ is exactly the set of points $\left(q_{l-1}, x_{l}\right) \in \mathcal{C}^{l}(m)$ such that $\mathcal{A}_{l-1}\left(q_{l-1}, x_{l}\right)=0$.

Now, if $l+1 \leq k$, then $q_{l+1}$ cannot be vertical and the point $q_{l+1}$ is no more regular, since otherwise the existence of a vertical point between $q_{l+1}$ and $q$ would contradict the definition of $l$. It follows that $q_{l+1}$ must be a tangency point.

We proceed by induction. Assume that for $l \leq i<k$ the point $q_{i}$ is tangency. By the same arguments as previously, $q_{i+1}$ must also be a tangency point. It follows that, by induction, we obtain (1).

We shall now prove (2). For each $0 \leq i \leq j \leq k-1$ we set

$$
\mathcal{A}_{j, i}\left(x_{0}, \ldots, x_{k}\right)=\left\langle x_{j+1}-x_{j}, x_{i+1}-x_{i}\right\rangle .
$$

Note that $\mathcal{A}_{j, i}$ is defined on any $\mathcal{C}^{h+l+1}(m)$ for $0 \leq i \leq j \leq h+l$.

For $l=0$, the point $q_{h+1}$ is vertical if and only if $\mathcal{A}_{h+1, h}\left(q_{h+1}\right)=0$, i.e., if and only if $q_{h+1}$ belongs to $\mathcal{C}_{q_{h}}^{h+1}(m-1)$ from Proposition 4.2(1). Therefore (2) is true for $l=0$. Assume that for all $0 \leq i<l$ the point $q_{h+i+1}$ belongs to $\mathcal{C}_{R^{h} V T^{i}}$ if and only if $\mathcal{A}_{j, h}\left(q_{h+j+1}\right)=0$ for all $j=h+1, \ldots, h+i$. By definition, the point $q_{h+l+1}$ belongs to $\mathcal{C}_{R^{h} V T^{l}}$ if and only if $q_{h+l+1}$ is tangency. From (1) each point $q_{h+i+1}$ under $q_{h+l+1}$ must be tangency for $i=0, \ldots, l-1$. In particular this means that $q_{h+l}$ belongs to $\mathcal{C}_{q_{h}}^{h+l}(m-1)$. It follows that $q_{h+l+1}$ is tangency if and only if the direction generated by $x_{h+l+1}-x_{h+l}$ belongs to $\delta_{l}^{h}\left(q_{h+l}\right)$ and according to the proof of Proposition 4.2 we get the relation $\left\langle x_{h+l+1}-x_{h+l}, x_{h+1}-x_{h}\right\rangle=0$. Therefore if $q_{h+l}$ belongs to $\mathcal{C}_{R^{h} V T^{l-1}}$ then $q_{h+l+1}$ belongs to $\mathcal{C}_{R^{h} V T^{l}}$ if and only if $\mathcal{A}_{h+l, h}\left(q_{h+l+1}\right)=0$.

$\mathcal{C}_{R^{h} V T^{l}}$ then is defined by the equations

$$
\mathcal{A}_{j, h}=0, \quad j=h+1, \ldots, h+l,
$$

in $\mathcal{C}^{h+l+1}(m)$. It is clear that these equations are independent. In particular, $q_{h+i+1}$ belongs to $\mathcal{C}_{q_{h}}^{h+i+1}(m-1)=F^{h+i+1}\left(\hat{P}^{i}\left(\hat{S}^{h+1}\left(q_{h}\right)\right)\right.$ for all $i=0, \ldots, k-h-l-1$. This ends the proof of $(2)$.

(3) is an interpretation of the equations (4.7) in terms of orthogonality.

\section{Relation between EKR classes of depth at most 1, RVT codes and articulated arms}

\subsection{Mormul EKR coding according to [8, 9]}

In $[8,9]$, P. Mormul has constructed a coding system for labeling singularity classes of germs of special multi-flag which he called "extended Kumpera-Ruiz" ("EKR" in short). Mormul's codes are finite sequences in $\mathbb{N}$. We now summarize how P. Mormul defines this coding system.

Given a coordinate system $\left(y^{1}, \ldots, y^{s}\right)$ on $\mathbb{R}^{s}$ consider a distribution $\mathcal{D}$ defined on a neighbourhood of $0 \in \mathbb{R}^{s}$ by $m+1$ vector fields $Z_{1}, \ldots, Z_{m+1}$. A new distribution $\mathcal{D}^{\prime}$ is associated with $\mathcal{D}$ on a neighbourhood of $0 \in \mathbb{R}^{s+m}$ relatively to a coordinate system $\left(y^{1}, \ldots, y^{s}, x^{1}, \ldots, x^{m}\right)$ by an operation denoted $\mathbf{j}$ where $\mathbf{j}$ takes values in $\{1,2, \ldots, m+1\}$ in the following way: for a fixed value $j$ of $\mathbf{j}$, the distribution $\mathcal{D}^{\prime}$ is generated by

$$
\begin{aligned}
& \left\{Z_{1}^{\prime}=x^{1} Z_{1}+\cdots+x^{j-1} Z_{j-1}+Z_{j}+\left(x^{j}+c^{j}\right) Z_{j+1}+\cdots+\left(x^{m}+c^{m}\right) Z_{m+1},\right. \\
& \left.Z_{2}^{\prime}=\frac{\partial}{\partial x^{1}}, \ldots, Z_{m+1}^{\prime}=\frac{\partial}{\partial x^{m}}\right\},
\end{aligned}
$$

where $c^{j}, \ldots, c^{m}$ are constants that may or may not be equal to zero. 
For instance, when $m=2, \mathcal{D}^{\prime}$ is a distribution of rank 3 generated by

$$
Z_{1}^{\prime}= \begin{cases}Z_{1}+\left(x^{1}+c^{1}\right) Z_{2}+\left(x^{2}+c^{2}\right) Z_{3} & \text { for } \mathbf{j}=1 \\ x^{1} Z_{1}+Z_{2}+\left(x^{2}+c^{2}\right) Z_{3} & \text { for } \mathbf{j}=2, \\ x^{1} Z_{1}+x^{2} Z_{2}+Z_{3} & \text { for } \mathbf{j}=3\end{cases}
$$

and $Z_{2}^{\prime}=\frac{\partial}{\partial x^{1}}, Z_{3}^{\prime}=\frac{\partial}{\partial x^{2}}$.

This procedure is initiated for $\mathcal{D}^{(0)}$ generated by $\left\{Z_{0}^{(0)}=\frac{\partial}{\partial y^{1}}, \ldots, Z_{m+1}^{(0)}=\frac{\partial}{\partial y^{m+1}}\right\}$ on $\mathbb{R}^{m+1}$ and we obtain by a first operation $\mathbf{j}_{1}$ a new distribution $\mathcal{D}^{(1)}$ of rank $m+1$ on a neighborhood of $0 \in \mathbb{R}^{2 m+1}$ generated by the produced vector fields $\left\{Z_{1}^{(1)}, \ldots, Z_{m+1}^{(1)}\right\}$. By induction on the composition of consecutive operations $\mathbf{j}_{\mathbf{1}}, \mathbf{j}_{\mathbf{2}}, \ldots, \mathbf{j}_{\mathbf{k}}$ for each word $\mathbf{j}_{\mathbf{1}} \mathbf{j}_{\mathbf{2}} \ldots \mathbf{j}_{\mathbf{k}}$, we obtain a distribution $\Delta_{\mathbf{j}_{1} \ldots \mathbf{j}_{\mathbf{k}}}$ on a neighborhood of $0 \in \mathbb{R}^{(k+1) m+1}$ generated by the associated produced $(m+1)$ vector fields $\left\{Z_{1}^{(k)}, Z_{2}^{(k)}, \ldots, Z_{m+1}^{(k)}\right\}$.

We have then the following result:

Theorem 5.1 (see $[8,9]$ ). Consider a differential system $D$ which spans a special multi-flag on a manifold $M$ of dimension $(k+1) m+1$. Every point $x \in M$ the differential system $(M, D, x)$ is locally equivalent to some differential system $\left(\Delta_{\mathbf{j}_{1} \mathbf{j}_{2} \ldots \mathbf{j}_{\mathbf{k}}}, \mathbb{R}^{(k+1) m+1}, 0\right)$. Moreover, the value of $\mathbf{j}_{\mathbf{1}} \mathbf{j}_{\mathbf{2}} \ldots \mathbf{j}_{\mathbf{k}}$ can be chosen such that $\mathbf{j}_{\mathbf{1}}=1$ and, in the case where $\mathbf{j}_{\mathbf{1}+\mathbf{1}}>\max \left(\mathbf{j}_{\mathbf{1}}, \mathbf{j}_{\mathbf{2}}, \ldots, \mathbf{j}_{\mathbf{1}}\right)$ then we have $\mathbf{j}_{\mathbf{1}+\mathbf{1}}=1+\sup \left(\mathbf{j}_{\mathbf{1}}, \mathbf{j}_{\mathbf{2}}, \ldots, \mathbf{j}_{\mathbf{l}}\right)$ for $l=1, \ldots, k-1$ (the rule of the least possible new jumps upwards).

Therefore, with a given germ of distribution $(M, D, x)$ on a manifold $M$ it is associated a well defined sequence of values $j_{1} j_{2} \ldots j_{k}$ which satisfies the rule of least upward jumps. Conversely, a germ of distribution $D$ determines an unique sequence of operations $\mathbf{j}_{\mathbf{1}}, \mathbf{j}_{\mathbf{2}}, \ldots, \mathbf{j}_{\mathbf{k}}$ satisfying the rule of least upward jumps (see footnote 6 of [9]). This sequence is called a singularity class of special multi-flags in [8]. We will say that this a sequence is an EKR class of germ of distributions which is encoded by the unique associated sequence of integers $j_{1} j_{2} \ldots j_{k}$.

Since $\mathcal{D}_{k}$ generates a special multi-flag of step $m \geq 2$ and length $k \geq 1$ on $\mathcal{C}^{k}(m)$, it is associated with any point $q \in \mathcal{C}^{k}(m)$ a word $j_{1} j_{2} \ldots j_{k}$ defined by the germ $\left(\mathcal{D}_{k}, \mathcal{C}^{k}(m), q\right)$. We will denote by $\Sigma_{j_{1} j_{2} \ldots j_{k}}$ the set of configurations $q \in \mathcal{C}^{k}(m)$ such that this associated word is $j_{1} j_{2} \ldots j_{k}$ and called it an EKR set. The integer $d=\sup \left\{j_{1}, \ldots, j_{k}\right\}-1$ will be called the depth of $\Sigma_{j_{1} j_{2} \ldots j_{k}}$ (see Fig. 4).

\subsection{Stratification of EKR sets of depth at most 1 by RVT codes}

According to the notations introduced in Section 1, the following result gives a complete description of EKR sets of depth at most 1 in terms of RVT sets. This result gives a proof of Theorems 2 and $3(2)$ :

\section{Theorem 5.2.}

1. The EKR set $\Sigma_{1 \ldots 1}$ is the set of Cartan points. In particular, $\Sigma_{1 \ldots 1}$ is an open dense set whose complementary is a subanalytic set of $\mathcal{C}^{k}(m)$ of codimension 1.

2. Let $\Sigma_{j_{1} \ldots j_{k}}$ be an EKR set of depth 1 and $\left\{i_{1}, \ldots, i_{\nu}\right\}$ be the set of indices $i$ such that $j_{i}=2$. Then $\Sigma_{j_{1} \ldots j_{k}}$ is an analytic submanifold of $\mathcal{C}^{k}(m)$ of codimension $\nu$. Moreover, $q$ belongs to $\Sigma_{j_{1} \ldots j_{k}}$ if and only if the configuration at $q$ of the articulated arm $\left(M_{0}, \ldots, M_{k}\right)$, the segments $\left[M_{i-2}, M_{i-1}\right]$ and $\left[M_{i-1}, M_{i}\right]$ are orthogonal at $M_{i-1}$ for all index $i \in\left\{i_{1}, \ldots, i_{\nu}\right\}$.

3. In the previous situation we have 


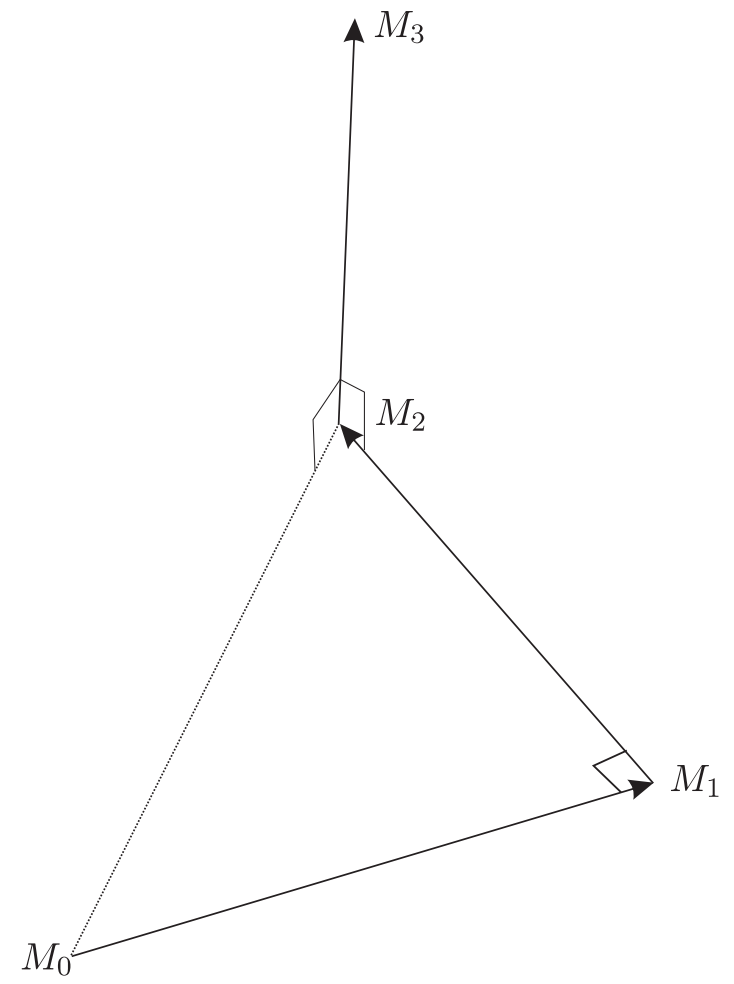

Figure 4. EKR set $\Sigma_{123}$ of depth 2 of an articulated arm $\left(M_{0}, M_{1}, M_{2}, M_{3}\right)$.

(i) A point $q$ belongs to $\Sigma_{j_{1} \ldots j_{k}}$ if and only if, in its $\mathbf{R V T}$ code the only letters $V$ are at rank $i_{1}, \ldots, i_{\nu}$.

(ii) A RVT set $\mathcal{C}_{\omega}$ is contained in $\Sigma_{j_{1} \ldots j_{k}}$ if and only if $\omega$ is of type $R^{h_{0}} V T^{l_{1}} R^{h_{1}} \ldots V T^{l_{\nu}} R^{h_{\nu}}$ and each letter $V$ is exactly at rank $i_{1}, \ldots, i_{\nu}$. More precisely, $l_{\lambda}+h_{\lambda}=i_{\lambda+1}-i_{\lambda}-1$ for $\lambda=1, \ldots, \nu-1$ and $l_{\nu}+h_{\nu}=k-i_{\nu}-1$. This set is an analytic submanifold of $\Sigma_{j_{1} \ldots j_{k}}$ of codimension $l_{1}+\cdots+l_{\nu}$. In particular $\mathcal{C}_{R^{h_{0} V R^{h_{1}}} \ldots V R^{h_{\nu}}}$ is an open dense set of $\Sigma_{j_{1} \ldots j_{k}}$ for $h_{0}=i_{1}-1, h_{\lambda}=i_{\lambda+1}-i_{\lambda}-1$ for $\lambda=1, \ldots, \nu-1$ and $h_{\nu}=k-i_{\nu}-1$.

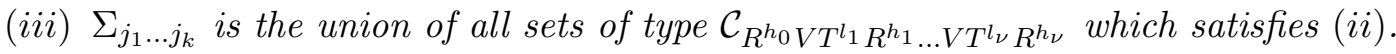

Remark 5.1. The decomposition of $\Sigma_{j_{1} \ldots j_{k}}$ given in (iii) above into RVT sets is in agreement with the decomposition of such EKR sets for $k=3$ described by Howard in the appendix of [4]. Therefore, the description in (iii) can be seen as a generalization of Howard's result.

For the proof of this theorem we need the following proposition which will be used in Section 5.4.

Proposition 5.1. Consider a configuration $q$ which belongs to some EKR set $\Sigma_{j_{1} \ldots j_{k}}$ of depthd.

1. If $d \geq 2$, we have $j_{h} \geq 2$ if and only if $\mathcal{A}_{h-1}=0$.

2. If $d \geq 2$ there exists a rank $h$ such that $j_{h}=3$ and the letter of rank $h$ in the RVT code of $q$ is of type $T_{0 i}$ with $i \geq 1$.

3. Assume that the RVT code of $q$ is a word which contains a letter of type $T_{r s .}$. Then this word contains also a letter of type $T_{0 i}$ for some rank $l<h$ with $i \geq 1$. If the letter of rank $h$ is $T_{0 i}$ with $i \geq 1$, then $2 \leq j_{h} \leq 3$. If $j_{h}=2$ there exists a letter of type $T_{01}$ at some rank $l<h$ with $j_{l}=3$. In particular $d \geq 2$.

4. If $d=2$, assume that the letter of rank $h$ is $T_{0 i}$ and $h$ is the first index $h$ such that $j_{h}=3$. Then $i=1$ and we have one and only one of the following situations: 
- $j_{h+1}=2$ if and only if the letter of rank $h+1$ is $V$ or $T_{02}$,

- $j_{h+1}=3$ if and only if the letter of rank $h+1$ is $T_{01}$.

Proof. According to the definition of the operation $\mathbf{j}$, a point $q_{l}$ is regular if and only if $j_{l}=1$, and according to the proof of Lemma 4.1, $q_{l}$ is regular if and only if $\mathcal{A}_{l-1}\left(q_{l}\right) \neq 0$. Therefore, since the depth of $\Sigma_{j_{1} \ldots j_{k}}$ is at most 2 , then $j_{l} \geq 2$ if and only if $\mathcal{A}_{l-1}=0$. This ends the proof of (1).

Now based on Proposition 4.2 and the convention in the RVT code, note that if the letter of rank $h$ is of type $T_{r s}$, there exists $\alpha<\beta$ such that the direction generated by $x_{h}-x_{h-1}$ belongs only the critical hyperplanes $\delta_{\alpha}^{h-\alpha-1}$ and $\delta_{\beta}^{h-\beta-1}$. Therefore from Proposition 4.2 the point $q_{h}$ satisfies the following constraints:

$$
\mathcal{A}_{l, h-\alpha-2}=0 \quad \text { for } \quad h-\alpha \leq l<h \quad \text { and } \quad \mathcal{A}_{l, h-\beta-2}=0 \quad \text { for } \quad h-\beta \leq l<h .
$$

Assume that there exists an index $h$ such that $j_{h} \geq 3$. The rule of least upward jumps assumes that there exists a rank $l<h$ such that $j_{l}=3$. Therefore we may assume that $j_{h}=3$. In this case, the point $q_{h}$ is critical and based on Lemma 4.1 we get $\mathcal{A}_{h-1}\left(q_{h}\right)=0$. The definition of the operation $\mathbf{j}$ implies that the projection of $X_{h}\left(q_{h}\right)$ on $\mathcal{C}^{h-1}(m)$ is contained in an hyperplane of $\delta_{0}^{h-1}$ at $q_{h-1}$. It follows that $q_{h}$ belongs to some critical manifold $\mathcal{C}_{q_{l}}^{h}(m-1)$ so that the point $q_{l+1}$ under $q_{h}$ is vertical. In particular we have $l \leq h-2$. Based on our our convention on the RVT code, the letter of rank $h$ is of type $T_{0 i}$ with $i \geq 1$. This ends the proof of (2).

Assume now that the letter of rank $h$ is $T_{r s}$. As we have already seen, there exists $\alpha<\beta$ such that the direction generated by $x_{h}-x_{h-1}$ belongs to $\delta_{\alpha}^{h-\alpha-1}$ and $\delta_{\beta}^{h-\beta-1}$. Equations (5.1) imply that the direction generated by $x_{h-\alpha}-x_{h-\alpha-1}$ belongs to $\delta_{0}^{h-\alpha-1}$ and $\delta_{\beta-\alpha}^{h-\beta-\alpha-1}$. Thus the letter of rank $h-\alpha$ is of type $T_{0 i}$ with $i \geq 1$. Let $\nu$ be the first rank such that the corresponding letter in the RVT code is of type $T_{0 i}$. From our conventions on the RVT code this letter must be $T_{01}$. Assume that the direction generated by $x_{\nu}-x_{\nu-1}$ belongs to $\delta_{0}^{\nu-1}$ and $\delta_{\gamma}^{\nu-\gamma-1}$.

From equations (5.1) for $\nu-\gamma<j \leq \nu$ and $q_{\nu-\gamma}$, the point $q_{j}$ must be tangency and $q_{\nu-\gamma}$ must be vertical. This implies that $j_{\nu-\gamma} \geq 2$. But from the choice of $\nu$ it follows that each letter of rank smaller than $\nu-1$ is of type $R, V, T$. It follows that $j_{\nu-\gamma}=2$. Therefore $q_{j}$ is tangency for $\nu-\gamma<j \leq \nu$ and the two points $q_{\nu-\gamma}$ and $q_{\nu}$ are vertical. According to the choice of $\nu$, the letter of rank $\nu-\gamma$ is $V$ and if $\gamma>1$ all letters of rank $\nu-\gamma<l<\nu$ are equal to $T$. Again from the choice of $\nu, j_{l}=1$ for $h-\nu<l<\nu$ if $\nu>1$. We get now, at point $q_{\nu-1}$ the two relations

$$
\left\langle x_{\nu}-x_{\nu-1}, x_{\nu-1}-x_{\nu-2}\right\rangle=0 \quad \text { and } \quad\left\langle x_{\nu}-x_{\nu-1}, x_{\nu-\gamma}-x_{\nu-\gamma-1}\right\rangle=0 .
$$

For a given fixed point $q_{h-1}$ we get two independent linear relations in $x_{\nu}-x_{\nu-1}$. Now from the choice of $\nu$, each $j_{l}$ belongs to $\{1,2\}$ for $1 \leq l<\nu$. Therefore from the definition of the operation $\mathbf{j}$ and the rule of least upward jumps it follows that $j_{\nu}=3$. This ends the proof of (3).

Finally assume that the letter of rank $h$ is $T_{01}$ and $h$ is the first index $h$ such that $j_{h}=3$. Note that if $\mathcal{A}_{h}\left(q_{h+1}\right)=0$, again from Theorem 4.3(1) this implies that there exists $\alpha>0$ such that the point $q_{h-\alpha}$ is vertical and $q_{j}$ is tangency for $h-\alpha<j \leq h$. In particular $q_{h}$ belongs to $\mathcal{C}_{q_{h-\alpha-1}}^{h}(m-1)$.

On the one hand assume that $j_{h+1}=2$. This condition is equivalent to $\mathcal{A}_{h}\left(q_{h+1}\right)=0$ and the projection of $X_{h+1}\left(q_{h+1}\right)$ onto $\mathcal{C}^{h}(m)$ must not be tangent to $\mathcal{C}_{q_{h-\alpha-1}}^{h}(m-1)$ at $q_{h}$ otherwise $j_{h+1}=3$. This implies that $q_{h+1}$ is vertical but the letter of rank $h+1$ can not be $T_{01}$ otherwise the projection of $X_{h+1}\left(q_{h+1}\right)$ onto $\mathcal{C}^{h}(m)$ must be tangent to $\mathcal{C}_{q_{h-\alpha-1}}^{h}(m-1)$ at $q_{h}$. Assume that the letter of rank $h+1$ is of type $T_{r s}$, of course we have $T_{r s} \neq T_{01}$. Now from the proof of (2), the letter of rank $h+1$ and the previous must be of type $T_{0 i}$ with $i \geq 1$. Taking into account our convention on the RVT code we get $T_{r s}=T_{02}$ 
Conversely if the letter of rank $h+1$ is $V$ then we get $j_{h+1}=2$. If this letter is $T_{02}$, this means that $\mathcal{A}_{h, h-2}\left(q_{h+1}\right) \neq 0$ but $\mathcal{A}_{h}\left(q_{h+1}\right)=0$. It follows that the projection of $X_{h+1}\left(q_{h+1}\right)$ onto $\mathcal{C}^{h}(m)$ is not tangent to $\mathcal{C}_{q_{h-\alpha-1}}^{h}(m-1)$ and so we have $j_{h+1}=2$.

On the other hand assume that $j_{h+1}=3$. This implies that $\mathcal{A}_{h}\left(q_{h+1}\right)=0$ and the letter of rank $h+1$ must be of type $T_{r s}$. Suppose that $T_{r s} \neq T_{01}$, by the same argument as previously, we obtain $T_{r s}=T_{02}$ which implies that $j_{h+1}=2$ and gives rise to a contradiction. Therefore we must have $T_{r s}=T_{01}$. Conversely if the letter of rank $h+1$ is $T_{01}$, as we have already seen, we have $j_{h+1}=3$.

Proof of Theorem 5.2. (1) and (2) are consequences of Propositions 5.1(1), 4.1 and Theorem 4.2(1). Now from the convention on the RVT code and Proposition 5.1(2) the RVT code of a point in an EKR set of depth 1 contains only letters in $\{R, V, T\}$. Therefore property (i) in (3) is a consequence of Propositions 4.1 and 5.1(1).

We now focus on property (ii). If $\omega$ is of type $R^{h_{0}} V T^{l_{1}} R^{h_{1}} \ldots V T^{l_{\nu}} R^{h_{\nu}}$ then the letters $V$ are exactly at rank $i_{1}, \ldots, i_{\nu}$ with the relations $l_{\lambda}+h_{\lambda}=i_{\lambda+1}-i_{\lambda}-1$ for $\lambda=1, \ldots, \nu-1$ and $l_{\nu}+h_{\nu}=k-i_{\nu}-1$, so the set $\mathcal{C}_{R^{h_{0}} V T^{l_{1}} R^{h_{1}} \ldots V T^{l_{\nu}} R^{h_{\nu}}}$ must be contained in the set $\Sigma_{j_{1} \ldots j_{k}}$ from (i). Consider any word $\omega$ in a RVT code such that each letter of rank $i_{1}, \ldots, i_{\nu}$ is $V$, and take any $q \in \mathcal{C}_{\omega} \subset \mathcal{C}^{k}(m)$. If $i_{2}=i_{1}+1$ the RVT code of $q_{i_{2}-1}$ is of type $R^{h_{0}} V T^{l_{1}} R^{h_{1}}$ with $l_{1}=h_{1}=0$. Assume now that $i_{2}-i_{1}>1$. If for all $i_{1}<j<i_{2}$ each point $q_{j}$ is regular and then the RVT code of $q_{i_{2}-1}$ is of type $R^{h_{0}} V T^{l_{1}} R^{h_{1}}$ with $l_{1}=0$. Now suppose that there exists some $q_{j}$ under $q$ with $i_{1}<j<i_{2}$ which is critical. The point $q_{j}$ must be tangency by Theorem 4.3(1), and, moreover, for $i_{1}<j^{\prime} \leq j$, the point $q_{j^{\prime}}$ must also be tangency. We set $l_{1}=\max \left\{j-i_{1} \mid q_{j}\right.$ tangency $\}$. Then, for $i_{1}+l_{1}<j<i_{2}$, the point $q_{j}$ must be regular otherwise from the previous argument $q_{j}$ must be tangency which contradicts the defintion of $l_{1}$. It follows that the RVT code of $q_{i_{2}-1}$ is of type $R^{h_{0}} V T^{l_{1}} R^{h_{1}}$. By induction on $1 \leq i \leq \nu$, the same arguments shows that $\omega$ must be of type $R^{h_{0}} V T^{l_{1}} R^{h_{1}} \ldots V T^{l_{\nu}} R^{h_{\nu}}$.

Finally, from the proof of Theorem $4.2(2)$, it follows that equations of $\mathcal{C}_{R^{h_{0}} V T^{l_{1}} R^{h_{1}} \ldots V T^{l_{\nu}} R^{h_{\nu}}}$ is the union of $\nu$ systems of type (4.7), and so we get a set of $\nu+l_{1}+\cdots+l_{\nu}$ independent equations. It follows that $\mathcal{C}_{R^{h_{0}} V T^{l_{1}} R^{h_{1}} \ldots V T^{l_{\nu}} R^{h_{\nu}}}$ is an analytic submanifold of $\mathcal{C}^{k}(m)$ of codimension $\nu+$ $l_{1}+\cdots+l_{\nu}$. On the other hand, the equations of $\Sigma_{j_{1} \ldots j_{k}}$ are $\mathcal{A}_{i_{\lambda}-1}=0$ for $\lambda=1, \ldots, \nu$. These equations are exactly the first equations of the $\nu$ systems of type (4.7) which define

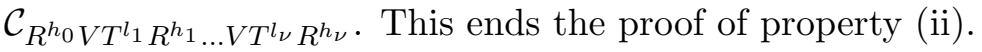

First of all from Proposition 5.1(3), if $d=1$ then the depth of any word in RVT code is 1. Therefore property (iii) is a direct consequence of properties (i) and (ii).

\subsection{EKR sets of depth 1, RVT codes and configurations of an articulated arms}

We will now give a complete interpretation of the previous result in terms of configurations of an articulated arm as stated in Theorem 3(2):

Theorem 5.3. Let $\Sigma_{j_{1} \ldots j_{k}}$ be an EKR set of depth 1 in $\mathcal{C}^{k}(m)$ and $\left\{i_{1}, \ldots, i_{\nu}\right\}$ the set $\{i \in$ $\left.\{2, \ldots, k\} \mid j_{i}=2\right\}$.

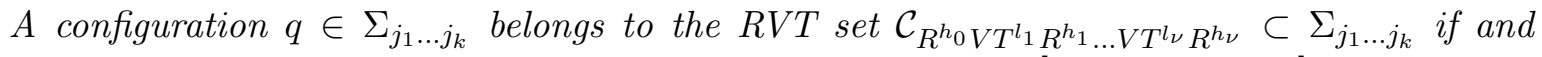
only, at $q$, the only orthogonality constraint is that each segment $\left[M_{i_{\lambda}+l-1}, M_{i_{\lambda}+l}\right]$ is orthogonal to the direction on $\mathbb{R}^{m+1}$ generated by $\overrightarrow{M_{i_{\lambda}-2} M_{i_{\lambda}-1}}$ for all $l=0, \ldots, l_{\lambda}$ and $\lambda=1, \ldots, \nu$.

Remark 5.2. The property (ii) of Theorem 4.3 is a particular case of Theorem 5.1. Note that we can find a similar result in [15] with more restricted context.

For the proof of this result, we need the notion of "induced articulated arm". 
Given two integers $r$ and $s$ such that $0 \leq r<s \leq k$, we can look for the motion of an induced articulated arm, which consists of segments of the original articulated arm joining $M_{r}$ to $M_{s}$. We can then study the induced articulated arm $\left(M_{r}, \ldots, M_{s}\right)$. We define $\kappa=s-r$, and we denote by $\mathcal{C}^{r s}(m)$ the image of $\mathcal{C}^{k}(m)$ by the canonical projection $\varrho^{r s}$ from $\mathbb{R}_{0}^{m+1} \times \cdots \times \mathbb{R}_{k}^{m+1}$ onto $\mathbb{R}_{r}^{m+1} \times \cdots \times \mathbb{R}_{s}^{m+1}$.

In fact, we have: $\mathcal{C}^{r s}(m)=\left\{q_{r s}=\left(x_{r}, x_{r+1}, \ldots x_{s}\right) \mid q=\left(x_{0}, \ldots, x_{k}\right) \in \mathcal{C}^{k}(m)\right\}$.

Taking into account Section 3.1 , let $\mathcal{E}_{r s}$ be the distribution on $\left(\mathbb{R}^{m+1}\right)^{\kappa+1}$ spanned by

$$
\mathcal{Z}_{r}, \quad \ldots, \quad \mathcal{Z}_{s-1}, \quad \frac{\partial}{\partial x_{s}^{1}}, \quad \ldots, \quad \frac{\partial}{\partial x_{s}^{m+1}}
$$

and let $\mathcal{D}_{r s}$ be the distribution induced by $\mathcal{E}_{r s}$ on $\mathcal{C}^{r s}(m)$.

In terms of Notations 3.2, the mechanical system describing the evolution of an induced arm $\left(M_{r}, \ldots, M_{s}\right)$ is a controlled system on $\mathbb{R}^{m+1} \times\left(\mathbb{S}^{m}\right)^{\kappa} \equiv \mathcal{C}^{\kappa}(m)$ naturally associated with the distribution $\mathcal{D}_{r s}$.

Consider a word $R^{h_{0}} V T^{l_{1}} R^{h_{1}} \ldots V T^{l_{\nu}} R^{h_{\nu}}$ of $k$ letters in a RVT code, and associate with this word the sequences $r_{0}, \ldots, r_{\nu}$ and $s_{0}, \ldots, s_{\nu}$ defined by

- $s_{0}=h_{0}$ and $r_{0}=0$,

- $s_{i}=s_{i-1}+h_{i}+l_{i}+1=h_{0}+h_{1}+l_{1}+1+\cdots+h_{i}+l_{i}+1$ and $r_{i}=s_{i-1}-1$ for $i=1, \ldots, \nu$.

We get then the following characterization:

Lemma 5.1. The configuration $q \in \mathcal{C}^{k}(m)$ of an articulated arm $\left(M_{0}, \ldots, M_{k}\right)$ belongs to the set $\mathcal{C}_{R^{h_{0}} V T^{l_{1}} R^{h_{1}} \ldots V T^{l_{\nu}} R^{h_{\nu}}}$ if and only if the induced articulated arm associated with the pair of integers $\left(r_{i}, s_{i}\right)$ is such that $\varrho^{r_{i} s_{i}}(q)$ belongs to the set $\mathcal{C}_{R^{h_{0}}} \subset \mathcal{C}^{r_{0} s_{0}}(m)=\mathcal{C}^{s_{0}}(m)$ for $i=0$ and belongs to the set $\mathcal{C}_{R V T^{h_{i}} R^{h_{i}}} \subset \mathcal{C}^{r_{i} s_{i}}(m)$ for all $i=1, \ldots, \nu$.

Proof. For any $q \in \mathcal{C}^{k}(m)$ we denote as usual by $q_{l}$ any point of $\mathcal{C}^{l}(m)$ under $q$, and we fix a configuration $q \in \mathcal{C}^{k}(m)$ of the articulated arm $\left(M_{0}, \ldots, M_{k}\right)$. First of all, for $i=0$, the induced articulated arm associated with $\left(r_{0}, s_{0}\right)$ has the induced configuration $q_{s_{0}}$. The RVT code of $q_{s_{0}}$ consists of $h_{0}$ first letters of the RVT code of $q$. Therefore, these first $h_{0}$ letters are $R^{h_{0}}$ if and only if the RVT code of $q_{s_{0}}$ is $R^{h_{0}}$.

Assume that the first $s_{i}$ letters of the RVT code of $q$ are $R^{h_{0}} V T^{l_{1}} R^{h_{1}} \ldots V T^{l_{i}} R^{h_{i}}$ if and only if the RVT code of the configuration $q_{r_{i} s_{i}}=\varrho^{r_{i} s_{i}}(q)$ of the associated induced articulated arm is $R^{h_{0}}$ for $i=0$ and $R V T^{l_{i}} R^{h_{i}}$ for all $1 \leq i \leq \mu-1<\nu$. Consider the configuration $q_{r_{\mu} s_{\mu}}=\varrho^{r_{\mu} s_{\mu}}(q) \in \mathcal{C}^{r_{\mu} s_{\mu}}(m)$ of the associated induced articulated arm. Denote by $q_{r_{\mu} l}$ the configuration under $q_{r_{\mu} s_{\mu}}$ for $r_{\mu} \leq l \leq s_{\mu}$, and set $\kappa_{\mu}=s_{\mu}-r_{\mu}$. By convention, the RVT code of $q_{r_{\mu} r_{\mu}+1}$ is $R$. Now, according to Proposition 4.1, $q_{r_{\mu} r_{\mu}+2}$ is vertical in $\mathcal{C}^{r_{\mu} s_{\mu}}(m) \equiv \mathcal{C}^{\kappa_{\mu}}(m)$ if and only if

$$
\sum_{j=1}^{m+1}\left(x_{r_{\mu}+2}^{j}-x_{r_{\mu}+1}^{j}\right)\left(x_{r_{\mu}+1}^{j}-x_{r_{\mu}}^{j}\right)=0 .
$$

This is equivalent to $\mathcal{A}_{r_{\mu}+1}(q)=0$. It follows that $q_{r_{\mu} r_{\mu+2}}$ is vertical if and only if $q_{r_{\mu+2}}$ is vertical. Finally, the letter of rank 2 in the RVT code of $q_{r_{\mu} s_{\mu}}$ is $V$ if and only if the letter of rank $s_{\mu}+1=h_{0}+h_{1}+l_{1}+1+\cdots+h_{\mu}+l_{\mu}+2$ is also $V$. Consider now an integer $r_{\mu}+2+l$ with $0 \leq l \leq l_{\mu}$. Either $q_{r_{\mu} r_{\mu}+l+2}$ is critical or it is regular. If $q_{r_{\mu} r_{\mu}+l+2}$ were vertical, then, from the previous argument, $q_{r_{\mu}+l+2}$ would also be vertical, which contradicts the definition of the set $\left\{i_{1}, \ldots, i_{\nu}\right\}$. Assume that $q_{r_{\mu} r_{\mu}+l+2}$ is tangency. Then $q_{r_{\mu} r_{\mu}+l^{\prime}+2}$ is also tangency for all $0 \leq l^{\prime} \leq l$. We have this property if and only if the following relations hold (see 4.7):

$$
\left\langle x_{r_{\mu}+l^{\prime}+3}-x_{r_{\mu}+l^{\prime}+2}, x_{r_{\mu}+1}-x_{r_{\mu}}\right\rangle=0 \quad \text { for all } 0 \leq l^{\prime} \leq l .
$$


According to our assumption and the equations of $\mathcal{C}_{R^{h_{0}} V T^{l_{1}} R^{h_{1} \ldots V T^{l} \mu_{-1}} R^{h_{\mu-1}}}$ (see proof of property (ii) in Theorem 5.2), $q_{r_{\mu} r_{\mu+l+2}}$ is tangency if and only if $q_{r_{\mu}+l+2}$ is also tangency.

Now, $q_{r_{\mu} r_{\mu+l+2}}$ is regular if and only if $\left\langle x_{r_{\mu}+l+3}-x_{r_{\mu}+l+2}, x_{r_{\mu}+1}-x_{\mu}\right\rangle \neq 0$. On the one hand, if $q_{r_{\mu}+l+2}$ is regular we must have $\left\langle x_{r_{\mu}+l+3}-x_{r_{\mu}+l+2}, x_{r_{\mu}+1}-x_{r_{\mu}}\right\rangle \neq 0$ and so $q_{r_{\mu} r_{\mu+l+2}}$ is regular. On the other hand, according to the choice of the set $\left\{i_{1}, \ldots, i_{\nu}\right\}$, if $q_{r_{\mu}+l+2}$ is critical then it must be tangency. From the definition of the RVT code, since $x_{r_{\mu}+l+3}-x_{r_{\mu}+l+2}$ belongs to one and only one critical hyperplane then $\left\langle x_{r_{\mu}+l+3}-x_{r_{\mu}+l+2}, x_{r_{\mu}+1}-x_{r_{\mu}}\right\rangle=0$ and therefore $q_{r_{\mu} r_{\mu}+l+2}$ can not be regular. $q_{r_{\mu} r_{\mu+l+2}}$ is regular if and only if $q_{r_{\mu+l+2}}$ is regular.

It follows that our assumption is then true for the integer $\mu$.

Proof of Theorem 5.3. Based on Theorem 4.3(3), for each induced articulated arm associated with a pair $\left(r_{i}, s_{i}\right)$, there exists a family of directions $\left\{K_{i}(q)\right\}$ in $\mathbb{R}^{m+1}$ for $q \in \mathcal{C}^{r_{i} s_{i}}(m)$ generated by $x_{r_{i}+1}-x_{r_{i}}$ such that the configuration $q_{r_{i}, s_{i}}$ belongs to the set $\mathcal{C}_{R^{V} T^{l_{i}} R^{h_{i}}} \subset \mathcal{C}^{r_{i} s_{i}}(m)$ if and only if this configuration fulfills the following property: each segment $\left[M_{r_{i}+1+l}, M_{r_{i}+2+l}\right]$ is orthogonal at $M_{r_{i}+1+l}$ to $K_{i}\left(q_{r_{i} s_{i}}\right)$, for $l=0, \ldots, l_{i}$ and there is no other orthogonality constraint.

The theorem is then a consequence of Lemma 5.1 .

\subsection{EKR sets of depth 2, RVT codes and configurations of an articulated arm for $1 \leq k \leq 4$}

The combination of all possible RVT codes of depth 2 has an exponential growth relatively to the length $k$ of a special multi-flag. Therefore, in this subsection we only describe the relations between EKR sets of 2-depth, RVT codes and configurations of articulated arms for $k=4$. In fact, this situation corresponds to the results of $[3,4,10]$.

First of all, for $k=3$, we have only $\Sigma_{123}$, which is an EKR set of depth 2, and for $k=4$ we have fourteen EKR sets (of depth 2) whose numerical codes are (see for instance [10]) 1111, 1112, 1121, 1122, 1123, 1211, 1212, 1213, 1221, 1222, 1223, 1231, 1232, 1233.

Therefore, for $1 \leq k \leq 4$, the other EKR sets for $1 \leq k \leq 4$ are of depth 1 .

Recall that at the end of Section 4.1 we have seen that for $k=3$ we have only one RVT set of depth 2 (i.e. $R T_{0} T_{01}$ ) but we have ten RVT sets for $k=4$. All other RVT sets are of depth at most 1.

Notice that the decomposition of EKR sets of depth 1 into RVT sets are of depth 1 can be found in Theorem 5.2, and the corresponding interpretation in terms of configurations of an articulated arm can also be found in Theorem 5.1. This is why we have given such results only for EKR sets of depth 2 previously enumerated.

For this purpose, we need the following characterizations of some EKR sets of depth 2 which is an easy consequence of Proposition 5.1:

Proposition 5.2. Let $\Sigma_{j_{1} \ldots j_{k}}$ be an EKR set of depth 2 in $\mathcal{C}^{k}(m)$ with $k \geq 3$. Consider an integer $2 \leq h<k$. Assume that $j_{l} \in\{1,2\}$ for all $1 \leq l \leq h-1$ and denote by $\left\{i_{1}, \ldots, i_{\nu}\right\}$ the set of indexes $i \in\{1, \ldots, h-1\}$ such that $j_{i}=2$.

1. $j_{h}=3$ if and only if the letter of rank $h$ of the RVT code of any $q \in \Sigma_{j_{1} \ldots j_{k}}$ is $T_{01}$ where $T_{1}$ refers to the singular hyperplane $\delta_{i_{\nu}}^{h-i_{\nu}-1}$.

2. if $j_{h}=3$ then we have one and only one of the following situations:

- $j_{h+1}=1$ if and only if the letter of rank $h+1$ belongs to the set $\left\{R, T_{1}, T_{2}, T_{12}\right\}$,

- $j_{h+1}=2$ if and only if the letter of rank $h+1$ belongs to the set $\left\{V, T_{02}\right\}$,

- $j_{h+1}=3$ if and only if the letter of rank $h+1$ is $T_{01}$. 
For $k=4$, we can easily get the decomposition of an EKR set of depth at most 2 into RVT sets as given in the following table by application of the previous proposition. For $k \leq 3$, the results are particular cases of Theorem 5.2.

Decomposition of EKR classes into RVT classes

\begin{tabular}{|c|c|}
\hline EKR class & RVT class \\
\hline 1111 & $R R R R$ \\
\hline 1112 & $R R R V$ \\
\hline 1121 & $R R V R, R R V T$ \\
\hline 1122 & $R R V V$ \\
\hline 1123 & $R R T_{0} T_{01}$ \\
\hline 1211 & $R V R R, R V T R, R V T T$ \\
\hline 1212 & $R V R V, R V T V$ \\
\hline 1213 & $R T_{0} T_{1} T_{01}$ \\
\hline 1221 & $R V V R, R V V T$ \\
\hline 1222 & $R V V V$ \\
\hline 1223 & $R V T_{0} T_{01}$ \\
\hline 1231 & $R T_{0} T_{01} R, R T_{0} T_{01} T_{1}, R V T_{0} T_{01} T_{2}, R V T_{0} T_{01} T_{12}$ \\
\hline 1232 & $R T_{0} T_{01} V, R T_{0} T_{01} T_{02}$, \\
\hline 1233 & $R T_{0} T_{01} T_{01}$. \\
\hline
\end{tabular}

For $1 \leq k \leq 4$, we only give an interpretation of RVT sets in terms of configurations of articulated arm when the RVT code contains a letter of type $T_{i j}$. The other cases are particular cases of Theorem 5.1. The proof of the following descriptions are obtained from the decomposition of each EKR set in RVT sets given in the previous table and by an easy interpretation in terms of orthogonality of the equations of type $\mathcal{A}_{j, i}=0$ of each such sets (see the proof of Theorem 4.3). These proofs are left to the reader.

Let $q=\left(x_{0}, \ldots, x_{k}\right)$ be a configuration of an articulated arm $\left(M_{0}, \ldots, M_{k}\right)$ with $k \leq 4$. We have the following characterizations:

- $q$ belongs to $\Sigma_{123}=\mathcal{C}_{R T_{0} T_{01}}$ if and only if $\left[M_{i-2}, M_{i-1}\right]$ and $\left[M_{i-1}, M_{i}\right]$ are orthogonal at $M_{i-1}$ for $i=2,3,\left[M_{2}, M_{3}\right]$ is orthogonal to the direction generated by $\overrightarrow{M_{0} M_{1}}$ and no other orthogonality in the configuration $q$.

- $q$ belongs to $\Sigma_{1123}=\mathcal{C}_{R R T_{0} T_{01}}$ if and only if $\left[M_{i-2}, M_{i-1}\right]$ and $\left[M_{i-1}, M_{i}\right]$ are orthogonal at $M_{i-1}$ for $i=3,4,\left[M_{3}, M_{4}\right]$ is orthogonal to the direction generated by $\overrightarrow{M_{1} M_{2}}$ and no other orthogonality in the configuration $q$.

- $q$ belongs to $\mathcal{C}_{R T_{0} T_{1} T_{01}}=\Sigma_{1213}$ if and only if $\left[M_{i-2}, M_{i-1}\right]$ and $\left[M_{i-1}, M_{i}\right]$ are orthogonal at $M_{i-1}$ for $i=2,4,\left[M_{3}, M_{4}\right]$ is orthogonal to the direction generated by $\overrightarrow{M_{0} M_{1}}$ and no other orthogonality in the configuration $q$.

- $q$ belongs to $\Sigma_{1223}=\mathcal{C}_{R V T_{0} T_{01}}$ if and only if $\left[M_{i-2}, M_{i-1}\right]$ and $\left[M_{i-1}, M_{i}\right]$ are orthogonal at $M_{i-1}$ for $i=2,3,4,\left[M_{3}, M_{4}\right]$ is orthogonal to the direction generated by $\vec{M}_{0} M_{1}$ and no other orthogonality in the configuration $q$.

- in $\Sigma_{1231}$ :

(i) $q$ belongs to $\mathcal{C}_{R T_{0} T_{01} R}$ if and only if $\left[M_{i-2}, M_{i-1}\right]$ and $\left[M_{i-1}, M_{i}\right]$ are orthogonal at $M_{i-1}$ for $i=2,3,\left[M_{2}, M_{3}\right]$ is orthogonal to the direction generated by $\overrightarrow{M_{0} M_{1}}$ and no other orthogonality in the configuration $q$.

(ii) $q$ belongs to $\mathcal{C}_{R T_{0} T_{01} T_{1}}$ (resp. $\mathcal{C}_{R T_{0} T_{01} T_{2}}$ ) if and only if the previous constraints hold, $\left[M_{3}, M_{4}\right]$ is orthogonal to the direction generated by $\overrightarrow{M_{1} M_{2}}$ (resp. $\overrightarrow{M_{0} M_{1}}$ ) and no other orthogonality in the configuration $q$. 
(iii) $q$ belongs to $\mathcal{C}_{R T_{0} T_{01} T_{12}}$ if and only if we have the previous constraints of (ii) hold, $\left[M_{3}, M_{4}\right]$ is orthogonal at the directions generated by $\overrightarrow{M_{0} M_{1}}$ and by $\overrightarrow{M_{1} M_{2}}$ and no other orthogonality in the configuration $q$.

- in $\Sigma_{1232}$ :

(i) $q$ belongs to $\mathcal{C}_{R T_{0} T_{01} V}$ if and only if $\left[M_{i-2}, M_{i-1}\right]$ and $\left[M_{i-1}, M_{i}\right]$ are orthogonal at $M_{i-1}$ for $i=2,3,4,\left[M_{2}, M_{3}\right]$ is orthogonal to the directions generated $\overrightarrow{M_{0} M_{1}}$ and no other orthogonality in the configuration $q$.

(ii) $q$ belongs to $\mathcal{C}_{R T_{0} T_{01} T_{02}}$ if and only if $\left[M_{i-2}, M_{i-1}\right]$ and $\left[M_{i-1}, M_{i}\right]$ are orthogonal at $M_{i-1}$ for $i=2,3,4,\left[M_{2}, M_{3}\right]$ and $\left[M_{3}, M_{4}\right]$ are orthogonal at the direction generated by $\overrightarrow{M_{0} M_{1}}$ and $\overrightarrow{M_{1} M_{2}}$ respectively and no other orthogonality in the configuration $q$.

- The point $q$ belongs to $\mathcal{C}_{R T_{0} T_{01} T_{01}}=\Sigma_{1233}$ if and only if $\left[M_{i-2}, M_{i-1}\right]$ and $\left[M_{i-1}, M_{i}\right]$ are orthogonal at $M_{i-1}$ for $i=2,3,4,\left[M_{2}, M_{3}\right]$ and $\left[M_{3}, M_{4}\right]$ are orthogonal at the direction generated by $\overrightarrow{M_{0} M_{1}}$ and no other orthogonality in the configuration $q$.

Proof of Proposition 5.2. (1) is established in the proof of Proposition 5.1(3). In (2) the last two situations correspond to Proposition 5.1(4). The first situation is an elementary computation in terms of critical hyperplane and is left to the reader.

\section{Main notations}

- $\mathbb{D}: D=D_{k} \subset D_{k-1} \subset \cdots \subset D_{j} \subset \cdots \subset D_{1} \subset D_{0}=T M$ : special multi-flag of step $m \geq 2$ and length $k \geq 1$ (Section 2.1).

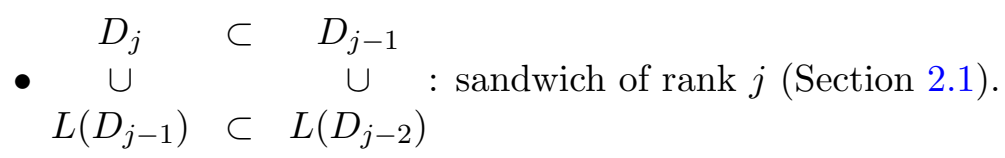

- $P^{k}(m) \rightarrow P^{k-1}(m) \rightarrow \cdots \rightarrow P^{1}(m) \rightarrow P^{0}(m):=\mathbb{R}^{m+1}$ : tower of projective bundles (Section 2.2).

- $\Delta_{j}$ : typical distribution on $P^{j}(m)$ which is the Cartan prolongation of $\Delta_{j-1}$ (Section 2.2).

- $S(D, M, g)$ : sphere bundle in the distribution $D$ associated with Riemannain metric $g$ (Section 2.3).

- $\hat{P}^{k}(m) \rightarrow \hat{P}^{k-1}(m) \rightarrow \cdots \rightarrow \hat{P}^{1}(m) \rightarrow \hat{P}^{0}(m):=\mathbb{R}^{m+1}$ : tower of sphere bundles (Section 2.3).

- $\hat{\Delta}_{j}$ : typical distribution on $\hat{P}^{j}(m)$ which is the spherical prolongation of $\hat{\Delta}_{j-1}$ (Section 2.3).

- $\left(M_{0}, \ldots, M_{k}\right)$ articulated arm or system of rigid bars in $\mathbb{R}^{m+1}$ of length $k$ (Section 3.1).

- $\mathcal{C}^{k}(m)$ : configuration space of an articulated arm in $\mathbb{R}^{m+1}$ of length $k$ (Section 3.1).

- $q=\left(x_{1}, \ldots, x_{k}\right) \in \mathcal{C}^{k}(m)$ : configuration of an articulated arm where $x_{i}=\left(x_{i}^{1}, \ldots, x_{i}^{r}, \ldots\right.$, $x_{i}^{m+1}$ ) for $i=0, \ldots, k$.

- $\mathcal{D}_{k}$ : typical distribution on $\mathcal{C}^{k}(m)$ associated with an articulated arm of length $k$ (Section 3.1).

- $\mathcal{Z}_{i}=\sum_{r=1}^{m+1}\left(x_{i+1}^{r}-x_{i}^{r}\right) \frac{\partial}{\partial x_{i}^{r}}$ for $i=0, \ldots, k-1$ (Section 3.1).

- $\mathcal{A}_{i}=\sum_{r=1}^{m+1}\left(x_{i+1}^{r}-x_{i}^{r}\right)\left(x_{i}^{r}-x_{i-1}^{r}\right)$ for $i=0, \ldots, k-1$ (Section 3.1). 
- $\mathcal{A}_{j, i}=\sum_{r=1}^{m+1}\left(x_{j+1}^{r}-x_{j}^{r}\right)\left(x_{i+1}^{r}-x_{i}^{r}\right)$ for $i=0, \ldots, k-1$ and $i<j<k$ (in proof of Theorem 4.3).

- $Y_{k}=\left(\sum_{i=0}^{k-2} \prod_{j=i+1}^{k-1} \mathcal{A}_{j} \mathcal{Z}_{i}\right)+\mathcal{Z}_{k-1}=\mathcal{A}_{k-1} Y_{k-1}+\mathcal{Z}_{k-1}$ (Section 3.1).

- $X_{k}=Y_{k}+\sum_{r=1}^{m+1}\left(x_{k}^{r}-x_{k-1}^{r}\right) \frac{\partial}{\partial x_{k}^{r}}($ Section 3.1).

- $\mathcal{D}_{k}$ is spanned by (Section 3.1):

$\circ\left\{\left(x_{k}^{r}-x_{k-1}^{r}\right) Y_{k}+\frac{\partial}{\partial x_{k}^{r}}, r=1, \ldots m+1\right\}$,

$\circ\left\{\left(x_{k}^{r}-x_{k-1}^{r}\right) X_{k}+\Pi_{k}\left(\frac{\partial}{\partial x_{k}^{r}}\right), r=1, \ldots m+1\right\}$,

$\circ\left\{X_{k}, \Pi_{k}\left(\frac{\partial}{\partial x_{k}^{r}}\right): r=1, \ldots, m+1\right\}$, where $\Pi_{k}: T\left(\mathbb{R}^{m+1}\right)^{k+1} \rightarrow T \mathcal{C}^{k}(m)$ is orthogonal projection.

- $\Psi^{j}: S\left(\mathcal{D}_{j}, \mathcal{C}^{j}(m), \gamma_{j}\right) \rightarrow \mathcal{C}^{j+1}(m)$ such that $\Psi_{*}^{j}\left(\left(\mathcal{D}_{j}\right)^{[1]}\right)=\mathcal{D}^{j+1}$ (Section 3.2).

- $F^{j}: \hat{P}^{j}(m) \rightarrow \mathcal{C}^{j}(m)$ such that $F_{*}^{j}\left(\hat{\Delta}_{j}\right)=\mathcal{D}_{j}$ (Section 3.2).

- $\mathcal{F}^{k}: \mathcal{C}^{k}(m) \rightarrow \mathcal{S}^{k}(m) \equiv \mathbb{R}^{m+1} \times\left(\mathbb{S}^{m}\right)^{k}$ with $\mathcal{F}^{k}\left(x_{0}, \ldots, x_{k}\right)=\left(x_{0}, x_{1}-x_{0}, \ldots, x_{k}-x_{k-1}\right)$ (Section 3.2).

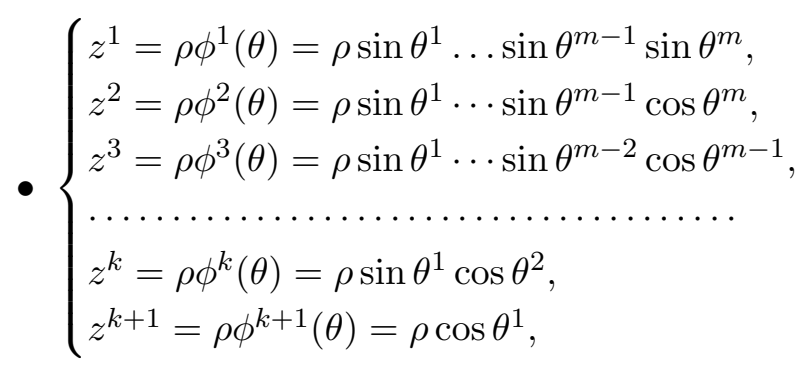

hyperspherical coordinates (Section 3.2).

- $\mathcal{P}^{j}(m)$ : either $P^{j}(m)$ or $\hat{P}^{j}(m)$ (Section 4.1).

- $\mathfrak{D}_{j}$ : the typical distribution on $\mathcal{P}^{j}(m)$ (i.e. either $\Delta_{j}$ or $\hat{\Delta}_{j}$ ) (Section 4.1).

- $\mathfrak{d}_{j}^{i}$ with $j+i=k$ : family of singular hyperplanes inside $\mathfrak{D}_{k}$ (Section 4.1).

- RVT code (Section 4.1).

- $\mathcal{C}_{\omega}$ : set of configurations $q \in \mathcal{C}^{k}(m)$ whose RVT code is the word $\omega$ (Section4.1).

- operation $\mathbf{j}$ (Section 5.1).

- EKR class (Section 5.1).

- $\Sigma_{j_{1} \ldots j_{k}}$ : set of configuration $q \in \mathcal{C}^{k}(m)$ for which the germ of the distribution $\mathcal{D}_{k}$ in $q$ belongs to the EKR class coded by $j_{1} \ldots j_{k}$.

\section{Acknowledgments}

We would like to thank warmly the anonymous referees for the care and time they spent in the detailed reading of different versions and for their questions, suggestions and comments that helped us to significantly improve the initial version. 


\section{References}

[1] Adachi J., Global stability of distributions of higher corank of derived length one, Int. Math. Res. Not. 2003 (2003), 2621-2638.

[2] Adachi J., Global stability of special multi-flags, Israel J. Math. 179 (2010), 29-56.

[3] Castro A.L., Howard W.C., A Monster Tower approach to Goursat multi-flags, Differential Geom. Appl. 30 (2012), 405-427.

[4] Castro A.L., Montgomery R., Spatial curve singularities and the Monster/Semple tower, Israel J. Math. 192 (2012), 381-427.

[5] Kumpera A., Rubin J.L., Multi-flag systems and ordinary differential equations, Nagoya Math. J. 166 (2002), 1-27.

[6] Li S.J., Respondek W., The geometry, controllability, and flatness property of the $n$-bar system, Internat. J. Control 84 (2011), 834-850.

[7] Montgomery R., Zhitomirskii M., Geometric approach to Goursat flags, Ann. Inst. H. Poincaré Anal. Non Linéaire 18 (2001), 459-493.

[8] Mormul P., Geometric singularity classes for special $k$-flags, $k \geq 2$, of arbitrary length, in Singularity Theory Seminar, Editor S. Janeczko, Warsaw University of Technology, Vol. 8, 2003, 87-100.

[9] Mormul P., Multi-dimensional Cartan prolongation and special $k$-flags, in Geometric Singularity Theory, Banach Center Publ., Vol. 65, Polish Acad. Sci., Warsaw, 2004, 157-178.

[10] Mormul P., Pelletier F., Special 2-flags in lengths not exceeding four: a study in strong nilpotency of distributions, arXiv:1011.1763.

[11] Pasillas-Lépine W., Respondek W., Contact systems and corank one involutive subdistributions, Acta Appl. Math. 69 (2001), 105-128, math.DG/0004124.

[12] Pasillas-Lépine W., Respondek W., On the geometry of Goursat structures, ESAIM Control Optim. Calc. Var. 6 (2001), 119-181, math.DG/9911101.

[13] Pelletier F., Espace de configuration d'un système mécanique et tours de fibrés associées à un multi-drapeau spécial, C. R. Math. Acad. Sci. Paris 350 (2012), 71-76.

[14] Shibuya K., Yamaguchi K., Drapeau theorem for differential systems, Differential Geom. Appl. 27 (2009), 793-808.

[15] Slayman M., Bras articulé et distributions multi-drapeaux, Ph.D. Thesis, Université de Savoie, Laboratoire de Mathématiques (LAMA), 2008.

[16] Slayman M., Pelletier F., Articulated arm and special multi-flags, J. Math. Sci. Adv. Appl. 8 (2011), 9-41, arXiv:1205.2990. 